Research Article

\title{
Investigation of Piezoelectric Energy Harvesting via Nonlinear Friction-Induced Vibration
}

\author{
D. W. Wang $\mathbb{D}^{1}{ }^{1}$ M. X. Liu, ${ }^{1}$ X. Wu, ${ }^{1}$ W. J. Qian, ${ }^{2}$ Q. Ma, ${ }^{1}$ and Z. Q. Wu ${ }^{1}$ \\ ${ }^{1}$ Science and Technology on Reactor System Design Technology Laboratory, Nuclear Power Institute of China, \\ Chengdu 610213, China \\ ${ }^{2}$ School of Mechatronic Engineering, Southwest Petroleum University, Chengdu 610500, China
}

Correspondence should be addressed to D. W. Wang; 1776135059@qq.com

Received 21 March 2020; Revised 14 June 2020; Accepted 9 July 2020; Published 27 July 2020

Academic Editor: Evgeny Petrov

Copyright (c) 2020 D. W. Wang et al. This is an open access article distributed under the Creative Commons Attribution License, which permits unrestricted use, distribution, and reproduction in any medium, provided the original work is properly cited.

In this work, piezoelectric energy harvesting (PEH) performance via friction-induced vibration (FIV) is studied numerically. A nonlinear two-degree-of-freedom friction system (mass-on-belt) with piezoelectric elements, which simultaneously considers the stick-slip motion, model coupling instability, separation, and reattachment between the mass and belt, is proposed. Both complex eigenvalue analyses and transient dynamic analysis of this nonlinear system are carried out. Results show that it is feasible to convert FIV energy to electrical energy when the friction system is operating in the unstable vibration region. There exists a critical friction coefficient $\left(\mu_{\mathrm{c}}\right)$ for the system to generate FIV and output visible voltage. The friction coefficient plays a significant role in affecting the dynamics and PEH performance of the friction system. The friction system is able to generate stronger vibration and higher voltage in the case that both the kinetic friction coefficient and static friction coefficient are larger than $\mu_{\mathrm{c}}$. Moreover, it is seen that the separation behavior between contact pair can result in overestimating or underestimating the vibration magnitude and output voltage amplitude, and the overestimate or underestimate phenomenon is determined by the located range of friction coefficient. Furthermore, it is confirmed that an appropriate value of external resistance is beneficial for the friction system to achieve the highest output voltage. The obtained results will be beneficial for the design of PEH device by means of FIV.

\section{Introduction}

Recently, with the problem of energy shortage becoming more and more serious, energy harvesting from ambient sources has become an important research field and received lots of attention from academia and industry [1-3]. Among the diverse new and renewable ambient energy sources, vibration, as a typical and common mechanical motion, has become an attractive source by virtue of its ubiquity and ease of access through MEMS technology. Over last decades, energy harvesting from ambient vibration has been extensively studied [4-7].

Generally, five main techniques can realize the conversion of vibration energy to electric energy, i.e., electromagnetic, electrostatic, piezoelectric, magnetostrictive, and triboelectric [8-14]. All these techniques have been successfully demonstrated experimentally and numerically in the past. An overview of the basics in energy harvesting has been provided by Priya et al. [3]. Among these techniques, piezoelectric energy harvesting (PEH) has attracted much more attention as it is easy to be implemented and does not rely on external voltage or magnetic field [15-20]. However, widespread usage of PEH technique is likely to be shadowed by a certain limitation; this limitation is mainly due to the fact that the natural frequency of PEH device is often not consistent with the vibration frequency from the ambient. As a matter of fact, an energy harvester can effectively implement its own functions only if ambient vibration frequency well matches with the resonant frequency of the harvester, which indicates that, with a slight deviation between these two frequencies, the ability of harvesters in energy harvesting would be reduced rapidly [6, 21-23]. Considering this, many new approaches, such as structure modification, circuit improvement, and nonlinear 
techniques, have been proposed to broaden the frequency response spectrum of harvester to match the ambient vibration frequency as much as possible [24-35]. However, most ambient vibrations are random and their frequency spectrums drift over time, which consequently brings about great challenges for the design of PEH devices [36].

To solve this problem, a new approach is proposed: seeking a kind of vibration energy which is independent of the ambient frequency and can be used for harvesting and conversion, such as friction-induced vibration (FIV). FIV is a typical self-excited vibration, which does not require any exterior force excitation, but it can be triggered under specific working conditions [37]. FIV phenomenon is commonly seen in our daily lives $[38,39]$. Examples include chatter or squeak from brake systems, tool cutting systems, mechanical gear systems, window wiper blades, and lead screw drives [40-42]. Considering that the FIV is uncontrollable and unpredictable due to its inherently irregular nature and it is undesirable and harmful in most cases; thus a large amount of research on FIV mainly focuses on understanding its mechanism and searching for potential effective approaches to improve FIV of friction systems [43-47]. Meanwhile, from another perspective, sometimes the FIV can be useful if this kind of vibration energy is collected in a certain way and converted to electrical energy, which can be thought of as "makes the best out of an undesirable situation" [48]. Furthermore, FIV occurs simultaneously in both the normal and tangential directions of the friction system, indicating that the vibration energy in both directions can be collected [8].

Up to now, many works have pointed out that the most probable reasons for the occurrence of FIV are stick-slip motion and model coupling phenomenon [49-51]. To study the energy harvesting performance related to the stick-slip motion, Helseth [52] established a hook and loop system and found that the stick-slip motion between hook and loop would cause the fluctuations of contact force. These force fluctuations could accordingly excite different energy harvesting systems and generate fluctuating electric signals. Tadokoro et al. [53] and Masuda and Sawai [54] experimentally verified the feasibility of $\mathrm{PEH}$ via stick-slip motion; moreover, Tadokoro et al. [53] also established a one-degreeof-freedom (DOF) mathematical model to simulate the $\mathrm{PEH}$ by means of stick-slip motion. On the other hand, to study the energy harvesting related to the model coupling instability, Wang et al. [8] experimentally and numerically studied the mode-coupling instability between a pad and disc and verified the feasibility of energy harvesting from high-amplitude FIV. By using a wobbling disc energy harvester, Heffel and Hagedorn [55] found that converting FIV energy to electrical energy could lead to a broadband applicability. In addition, in a recent work performed by Stender et al. [48], external forcing was verified to be able to pull part of the friction energy out of the system and thus make energy harvesting possible in certain parameter ranges. These studies are definitely necessary for realizing energy harvesting via FIV. However, the knowledge on this aspect is still limited, and the PEH behavior via FIV remains unknown and somehow mysterious. In our previous work to detect the PEH via FIV, a single-degree-of-freedom friction system is proposed to discuss the possible $\mathrm{PEH}$ via stick-slip vibration. Secondly, a two-degree-of-freedom friction system is used to discuss the possible $\mathrm{PEH}$ via model-coupling vibration. Visibly, these two parts are almost independent [56]. However, in a real world of FIV, the stick-slip motion and model coupling instability usually occur simultaneously, and sometimes separation and subsequent reattachment behaviors between the contact pair may appear as well during the friction process $[57,58]$, which indicates that a more specific and complicated mathematical model should be established, to reveal the relationship between energy harvesting and FIV numerically. This paper makes such a contribution.

In this work, the $\mathrm{PEH}$ performance by means of FIV is analyzed numerically. A nonlinear two-degree-of-freedom (DOFs) friction system (mass-on-belt) with piezoelectric elements, which simultaneously considers the stick-slip motion, model coupling, separation, and reattachment between the mass and belt, is proposed. Through introducing piezoelectric elements into the friction system, the energy harvesting behaviors along both the tangential and normal directions by means of FIV are verified. Moreover, the effects of friction coefficient and the separation behavior between contact pair on the dynamics and energy harvesting are studied. Finally, the dynamics behaviors of the friction system with different external electric resistances are analyzed, and the role of electric resistances in affecting $\mathrm{PEH}$ performance is revealed. Some conclusions on the piezoelectric energy harvesting related to FIV are presented.

\section{Theoretical Modelling}

A friction system with two DOFs is employed to study the $\mathrm{PEH}$ via FIV; this system is extended from the work of Tadokoro et al. [53]. In their work, a ball sample rubs against a sliding flat sample and stick-slip motion occurs; then a piezoelectric film is attached with the fixture of the test setup and successfully transfers the FIV energy to electric energy. Based on the test configuration, a one-degree-of-freedom friction system is proposed to investigate the $\mathrm{PEH}$ via stickslip friction motion. Inspired by this system, a more realistic two-DOF friction system which simultaneously accounts for the stick, slip, loss of contact, and reattachment between the contact pair due to mode-coupling is proposed in this work, as shown in Figure 1.

The slider $(m)$ rubs against a moving belt, which has a constant siding velocity $v_{0}$. A number of linear springs and dampers link with the slider, which are used to store and dissipate energy of the friction system. The spring $k_{1}$ and the damper $c_{1}$ connect with the slider along the tangential direction ( $x$ direction), while a damper $c_{2}$ connects with the slider along the normal direction ( $y$ direction). Additionally, piezoelectric elements named Piezo-1 and Piezo-2 (loaded with external electric resistance $R$ ) are, respectively, introduced into the friction system along the tangential and normal directions. These two piezo elements can respond to the vibration of the system through strain, so as to convert the vibration energy in both directions. A spring $k_{3}$ is linked 


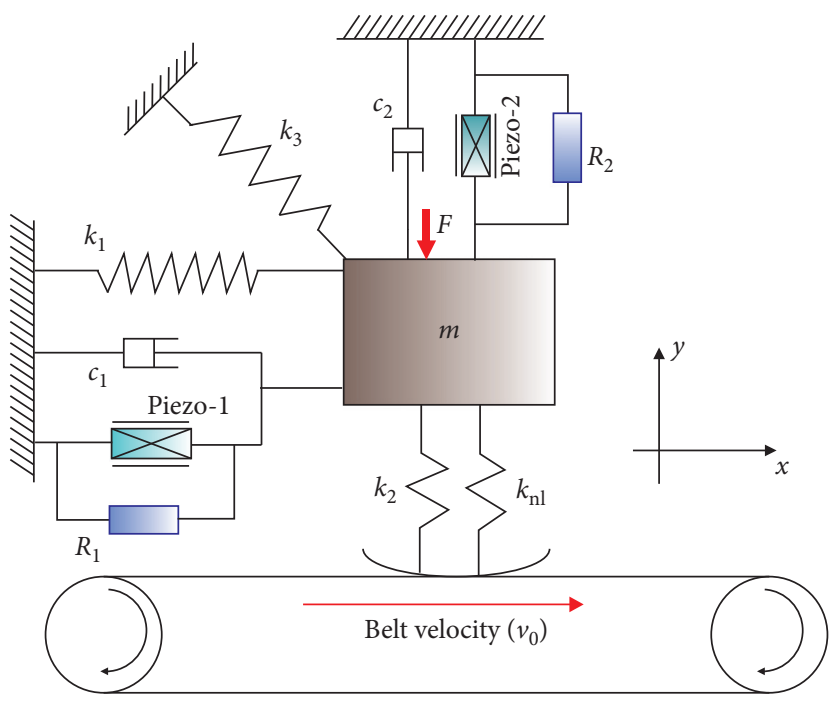

Figure 1: A 2-DOF friction system.

to the slider at a $135^{\circ}$ angle relative to the tangential direction, which provides coupling in the tangential and normal directions. Moreover, spring $k_{2}$ and $k_{\mathrm{n}}$, which are characterized by linear and cubic spring, respectively, are used to simulate the contact stiffness between contact pair. The effects of impact at reattachment between the slider and belt are neglected [57]. A constant normal compressive force, $F$, directly acts on the slider along the $y$ direction.
If the slider is still in contact with the belt, the contact force $F_{\mathrm{N}}$ between the contact pair is expressed as

$$
F_{N}=-k_{2} y-k_{n l} y^{3} \text {. }
$$

The equations of friction motion for the slider yield

$$
\begin{aligned}
& {\left[\begin{array}{cccc}
m & 0 & 0 & 0 \\
0 & m & 0 & 0 \\
0 & 0 & 0 & 0 \\
0 & 0 & 0 & 0
\end{array}\right]\left\{\begin{array}{c}
\ddot{x} \\
\ddot{y} \\
\ddot{V}_{x} \\
\ddot{V}_{y}
\end{array}\right\}+\left[\begin{array}{cccc}
c_{1} & 0 & 0 & 0 \\
0 & c_{2} & 0 & 0 \\
\alpha_{1} & 0 & -C_{p 1} & 0 \\
0 & \alpha_{2} & 0 & -C_{p 2}
\end{array}\right]\left\{\begin{array}{c}
\dot{x} \\
\dot{y} \\
\dot{V}_{x} \\
\dot{V}_{y}
\end{array}\right\}+\left[\begin{array}{cccc}
k_{1}+\frac{k_{3}}{2} & -\frac{k_{3}}{2}+\mu k_{2} & \alpha_{1} & 0 \\
-\frac{k_{3}}{2} & k_{2}+\frac{k_{3}}{2} & 0 & \alpha_{2} \\
0 & 0 & -\frac{1}{R_{1}} & 0
\end{array}\right]\left\{\begin{array}{c}
x \\
y \\
0 \\
V_{x} \\
V_{y}
\end{array}\right\}} \\
& +\left[\begin{array}{llll}
0 & \mu k_{n l} & 0 & 0 \\
0 & k_{n l} & 0 & 0 \\
0 & 0 & 0 & 0 \\
0 & 0 & 0 & 0
\end{array}\right]\left\{\begin{array}{c}
x^{3} \\
y^{3} \\
V_{x}^{3} \\
V_{y}^{3}
\end{array}\right\}=\left\{\begin{array}{c}
0 \\
-F \\
0 \\
0
\end{array}\right\} .
\end{aligned}
$$

In the above equation, the parameters $\alpha_{1}$ and $\alpha_{2}$ are defined as the force factors of Piezo-1 and Piezo-2, respectively; $V_{x}$ and $V_{y}$ represent the generated voltages from Piezo-1 and Piezo-2, respectively. It should be emphasized that equation (2) is valid only if the contact force still exists between contact pairs; that is,

$$
F_{N}(t)>0 .
$$

If $F_{N}(t)$ is found to be smaller than 0 , separation is identified to happen between the contact pair. The normal displacement of slider is larger than zero in this moment, and the springs $k_{2}$ and $k_{\mathrm{nl}}$ will not work on the contact stiffness. As a consequence, the situation where the slider separates with the belt can be depicted as shown in Figure 2, and the dynamical equation of the system can be expressed as the following equation: 


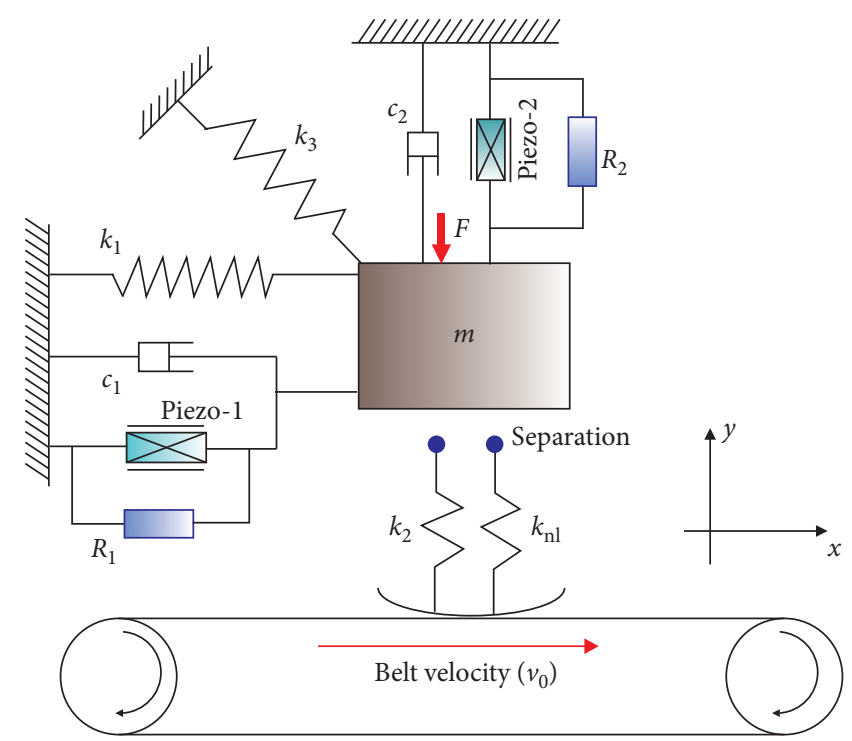

FIgURE 2: The situation where slider separates with the sliding belt.

$$
\left[\begin{array}{cccc}
m & 0 & 0 & 0 \\
0 & m & 0 & 0 \\
0 & 0 & 0 & 0 \\
0 & 0 & 0 & 0
\end{array}\right]\left\{\begin{array}{c}
\ddot{x} \\
\ddot{y} \\
\ddot{V}_{x} \\
\ddot{V}_{y}
\end{array}\right\}+\left[\begin{array}{cccc}
c_{1} & 0 & 0 & 0 \\
0 & c_{2} & 0 & 0 \\
\alpha_{1} & 0 & -C_{p 1} & 0 \\
0 & \alpha_{2} & 0 & -C_{p^{2}}
\end{array}\right]\left\{\begin{array}{c}
\dot{x} \\
\dot{y} \\
\dot{V}_{x} \\
\dot{V}_{y}
\end{array}\right\}+\left[\begin{array}{rrrr}
k_{1}+\frac{k_{3}}{2} & -\frac{k_{3}}{2} & \alpha_{1} & 0 \\
-\frac{k_{3}}{2} & \frac{k_{3}}{2} & 0 & \alpha_{2} \\
0 & 0 & -\frac{1}{R_{1}} & 0 \\
0 & 0 & 0 & -\frac{1}{R_{2}}
\end{array}\right]\left\{\begin{array}{c}
x \\
y \\
V_{x} \\
V_{y}
\end{array}\right\}=\left\{\begin{array}{c}
0 \\
-F \\
0 \\
0 \\
0 \\
0
\end{array}\right.
$$

Assuming that the belt surface is rigid, the condition of separation between the contact pair is determined by the normal displacement of slider, which is written as

$$
y(t)>0 .
$$

When the normal displacement $y(t)$ becomes less than zero, it is identified that the slider reattaches with the belt again. Then the dynamical equation of the friction is determined by equation (2) until the next round of separation appears again. Therefore, the velocity, displacement, and other state parameter values determined by equation (2) at the last step in contact are used for the initial conditions for equation (4), and the states of the mass before reattachment are the last moment in the separation motion (equation (2)).

\section{Eigenvalue Analysis}

It is meaningful to study the stability of the slider in the vicinity of the origin, which will be beneficial for detecting the critical friction coefficient $\mu_{c}$ for the generation of unstable vibration. In the eigenvalue analysis procedure, the slider is assumed to stay in the steady sliding stage; thus the separation and reattachment behaviors between the contact pair will not be accounted, and only (2) is employed to calculate the eigenvalue of the friction system.

The equilibrium points (EPs) $\left(x_{e}, y_{e}, V_{x e}\right.$, and $\left.V_{y e}\right)$ of the friction system are found by solving (2) in the case where the first and second derivatives of these four variables are assumed to be zero, which become

$$
\left[\begin{array}{cccc}
k_{1}+\frac{k_{3}}{2} & -\frac{k_{3}}{2}+\mu k_{2} & \alpha_{1} & 0 \\
-\frac{k_{3}}{2} & k_{2}+\frac{k_{3}}{2} & 0 & \alpha_{2} \\
0 & 0 & -\frac{1}{R_{1}} & 0 \\
0 & 0 & 0 & -\frac{1}{R_{2}}
\end{array}\right]\left\{\begin{array}{c}
x \\
y \\
V_{x} \\
V_{y}
\end{array}\right\}+\left[\begin{array}{cccc}
0 & \mu k_{n l} & 0 & 0 \\
0 & k_{n l} & 0 & 0 \\
0 & 0 & 0 & 0 \\
0 & 0 & 0 & 0
\end{array}\right]\left\{\begin{array}{c}
x^{3} \\
y^{3} \\
V_{x}^{3} \\
V_{y}^{3}
\end{array}\right\}=\left\{\begin{array}{c}
0 \\
-F \\
0 \\
0
\end{array}\right\} .
$$


Define the transformation relationships $z_{1}=x, z_{2}=\dot{x}, z_{3}=y, z_{4}=\dot{y}, z_{5}=V_{x}, z_{6}=V_{y}$. Thus equation (6) can be expressed in the following form:

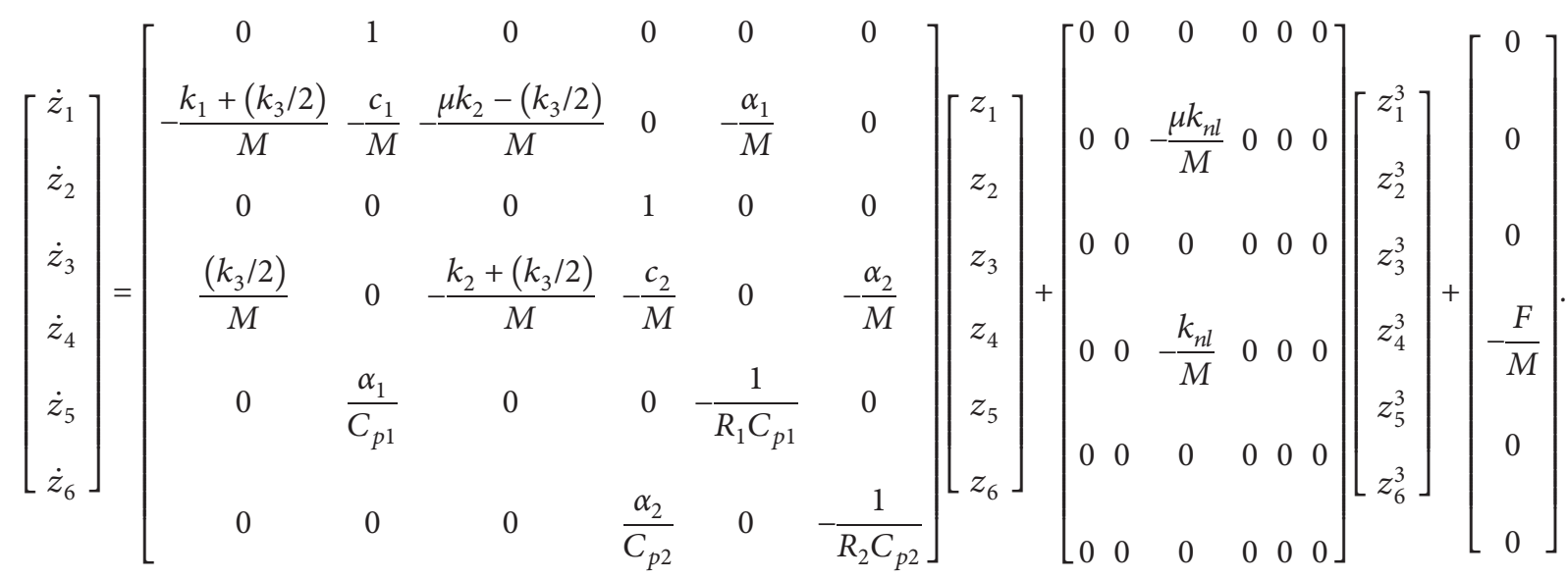

The Jacobian matrix can be derived via expanding the nonlinear equation (7) in a first-order truncated Taylor series at the equilibrium point, as written as follows:

$$
J=\left[\begin{array}{cccccc}
0 & 1 & 0 & 0 & 0 & 0 \\
-\frac{k_{1}+\left(k_{3} / 2\right)}{M} & -\frac{c_{1}}{M}-\frac{\mu k_{2}-\left(k_{3} / 2\right)}{M}-\frac{3 \mu k_{n l}}{M} y_{e}^{2} & 0 & -\frac{\alpha_{1}}{M} & 0 \\
0 & 0 & 0 & 1 & 0 & 0 \\
\frac{\left(k_{3} / 2\right)}{M} & 0 & -\frac{k_{2}+\left(k_{3} / 2\right)}{M}-\frac{3 k_{n l}}{M} y_{e}^{2} & -\frac{c_{2}}{M} & 0 & -\frac{\alpha_{2}}{M} \\
0 & \frac{\alpha_{1}}{C_{p 1}} & 0 & 0 & -\frac{1}{R_{1} C_{p 1}} & 0 \\
0 & 0 & 0 & \frac{\alpha_{2}}{C_{p 2}} & 0 & -\frac{1}{R_{2} C_{p 2}}
\end{array}\right] .
$$

The stability of the system can be evaluated by calculating the eigenvalues $\lambda$ of the Jacobian matrix [59]. The eigenvalues $\lambda$ of the matrix $[J]$ can be obtained by solving the following equation:

$$
\operatorname{det}(\lambda[E]-[J])=0 .
$$

The parameters set in this analysis are from the work performed by Tadokoro et al. [53], which are listed in Table 1. Figure 3 depicts the variation of real and imaginary parts of the eigenvalues with the increase of friction coefficients. Visibly, there exists a specified friction coefficient value $(0.85)$ at which system will become unstable. This value of friction coefficient is called critical friction coefficient $\mu_{c}$. The eigenvalues are a pair of complex conjugates with identical negative real parts for friction coefficient less than the critical value. At and beyond this value, the real parts of two eigenvalues branch out to one positive and one negative, while the corresponding imaginary parts start to merge into one value. Such mode merging phenomena indicate that the friction system has a strong tendency to generate self-excited instability, and accordingly the vibration energy may be transferred to electric energy through the action of Piezo elements. Based on the critical friction coefficient $\mu_{c}$, the nonlinear transient dynamic analysis of the friction system will be performed in the next section.

\section{Transient Dynamic Analysis}

4.1. Procedure of the Transient Dynamic Analysis. In the transient dynamic analysis, the stick, slip, separation, and reattachment behaviors between the slider and belt are taken 
TABLE 1: The parameters of the friction system set in the eigenvalues analysis.

\begin{tabular}{lccc}
\hline Mass (slider) & $0.08 \mathrm{~kg}$ & $k_{3}$ (coupling spring in two directions) & $600 \mathrm{~N} / \mathrm{m}$ \\
\hline$k_{1}$ (including the stiffness of Piezo-1) & $890 \mathrm{~N} / \mathrm{m}$ & $c_{1}=c_{2}$ (including the damping values of piezo elements) & $0.077 \mathrm{~N} /(\mathrm{m} / \mathrm{s})$ \\
$k_{n l}$ (nonlinear contact stiffness) & $1200 \mathrm{~N} / \mathrm{m}^{3}$ & $\alpha_{1}=\alpha_{2}$ (force factor) & $0.00036 \mathrm{~N} / \mathrm{V}$ \\
$k_{2}$ (linear contact stiffness) & $500 \mathrm{~N} / \mathrm{m}$ & $C_{p 1}=C_{p 2}$ (clamped capacitance of the piezo elements) & $1.79 \times 10^{-7} \mathrm{~F}$ \\
$v_{0}$ (belt velocity) & $5 \mathrm{~mm} / \mathrm{s} \sim 200 \mathrm{~mm} / \mathrm{s}$ & $F$ (normal load) & $20 \mathrm{~N}$ \\
$R_{1}$ & $10 \mathrm{k} \Omega$ & $R_{2}$ & $10 \mathrm{k} \Omega$ \\
\hline
\end{tabular}

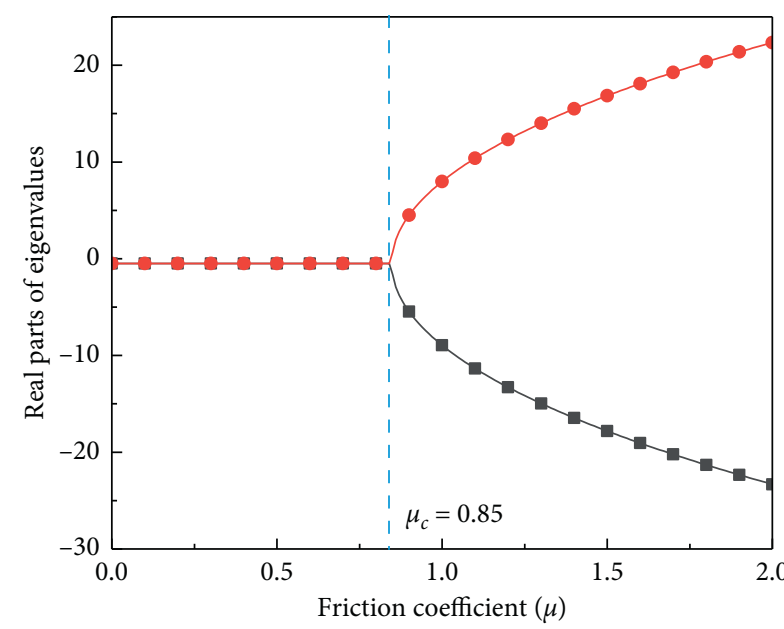

(a)

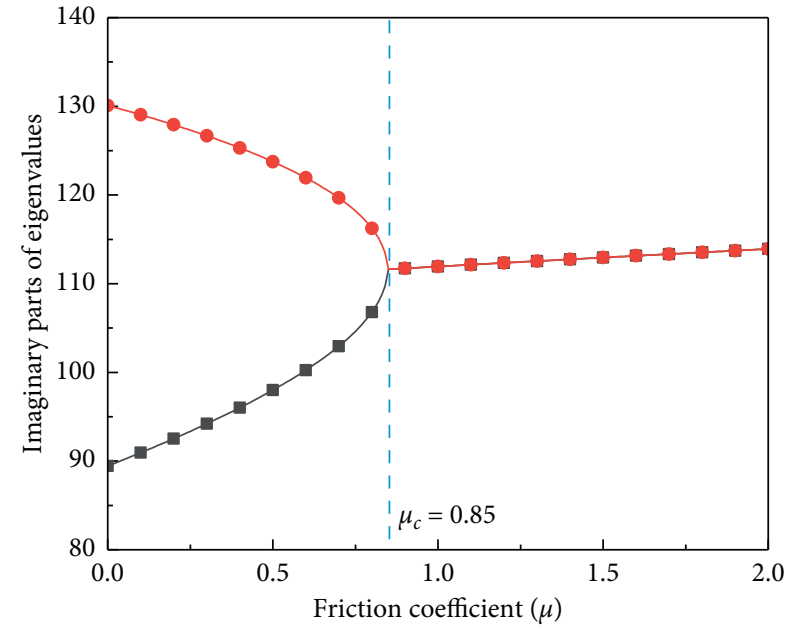

(b)

FIgURE 3: The real parts (a) and imaginary parts (b) of the friction system with the increase of friction coefficient.

into consideration. Assume that the relative sliding velocity $v_{r}$ is expressed as

$$
v_{r}=v_{0}-\dot{x}(t)
$$

Therefore, the friction system has two motion phases when the slider is sliding on the belt $(y<0)$, that is, slip phase $\left(v_{r} \neq 0\right)$ and stick phase $\left(v_{r}=0\right)$. For convenience of numerical computation, the criterion conditions for the relative velocity $v_{r} \neq 0$ and $v_{r}=0$ are changed to $\left|v_{r}\right|>\varepsilon$ and $\left|v_{r}\right| \leq \varepsilon$, respectively, where $\varepsilon$ represents a threshold value for the sliding velocity that is sufficiently close to zero $\left(10^{-6}\right)$.

When $\left|v_{r}\right|>\varepsilon$, that is, the friction system stays in the slip stage, the friction force $F_{f}$ is expressed as

$$
F_{f}=-\mu_{k}\left(k_{2} y(t)+k_{n l} y(t)^{3}\right) \operatorname{sgn}\left(v_{r}\right) .
$$

Here the parameter $\mu_{k}$ represents the kinetic friction coefficient of the friction system. When $\left|v_{r}\right| \leq \varepsilon$, it indicates that the friction system is in the stick stage; the friction force $F_{f}$ is determined by the maximum static friction force $F_{\text {static }}$ and the applied horizontal force $F_{\text {applied }}$ on the slider. Here $F_{\text {static }}$ is expressed as

$$
F_{\text {static }}=-\mu_{s}\left(k_{2} y(t)+k_{n l} y(t)^{3}\right) \operatorname{sgn}\left(v_{r}\right) .
$$

The applied horizontal force is expressed as

$$
F_{\text {applied }}=k_{1} x(t)+c_{1} \dot{x}(t)+\alpha V_{1}(t)+\frac{1}{2} k_{3} x(t)-\frac{1}{2} k_{3} y(t),
$$

and therefore, in the stick phase, the friction force $F_{f}$ can be summarized in the following form:

$$
F_{f}= \begin{cases}k_{1} x(t)+c_{1} \dot{x}(t)+\alpha_{1} V_{1}(t)+\frac{1}{2} k_{3} x(t)-\frac{1}{2} k_{3} y(t), & \text { when }\left|F_{\text {applied }}\right|<F_{\text {static }}, \\ -\mu_{s}\left(k_{2} y(t)+k_{n l} y^{3}(t)\right) \operatorname{sgn}\left(v_{r}\right), & \text { when }\left|F_{\text {applied }}\right|>F_{\text {static }} .\end{cases}
$$


Then equation (2) can be transformed to the following equation:

$$
\left[\begin{array}{c}
\dot{z}_{1} \\
\dot{z}_{2} \\
\dot{z}_{3} \\
\dot{z}_{4} \\
\dot{z}_{5}
\end{array}\right]=\left[\begin{array}{cccccc}
0 & 1 & 0 & 0 & 0 & 0 \\
-\frac{k_{1}+\left(k_{3} / 2\right)}{M} & -\frac{c_{1}}{M} & \frac{\left(k_{3} / 2\right)}{M} & 0 & -\frac{\alpha_{1}}{M} & 0 \\
0 & 0 & 0 & 1 & 0 & 0 \\
\frac{\left(k_{3} / 2\right)}{M} & 0 & -\frac{k_{2}+\left(k_{3} / 2\right)}{M} & -\frac{c_{2}}{M} & 0 & -\frac{\alpha_{2}}{M} \\
0 & \frac{\alpha_{1}}{C_{p 1}} & 0 & 0 & -\frac{1}{R_{1} C_{p 1}} & 0 \\
z_{2} \\
z_{3} \\
z_{2} \\
0
\end{array}\right.
$$

During sliding stage, the dynamical equation of the system is determined by equation (15), and the contact force between contact pair is estimated at the last time step; once the contact force $F_{N}<0$, it is suggested that the slider is separating with belt. At this moment, the equation of motion is changed to equation (4). The critical point is determined, which corresponds to the moment when the contact force $F_{N}=0$. At this critical point, the vibration state is switched from sliding stage to separation stage. During the separation process, the normal displacement of the slider $y(t)$ is detected, as long as $y(t)<0$, indicating that the slider is touching with the belt. The critical point $(y(t)=0)$ suggests the generation of reattachment between contact pair; at this moment, the equation of motion is changed to equation (2) until the stage of separation is satisfied again.

Equation (4) is used to calculate the dynamic behavior of the friction system in the separation process, which can be expressed as follows:

$$
\left[\begin{array}{c}
\dot{z}_{1} \\
\dot{z}_{2} \\
\dot{z}_{3} \\
\dot{z}_{4} \\
\dot{z}_{5} \\
\dot{z}_{6}
\end{array}\right]=\left[\begin{array}{cccccc}
0 & 1 & 0 & 0 & 0 & 0 \\
-\frac{k_{1}+\left(k_{3} / 2\right)}{M} & -\frac{c_{1}}{M} & \frac{\left(k_{3} / 2\right)}{M} & 0 & -\frac{\alpha_{1}}{M} & 0 \\
0 & 0 & 0 & 1 & 0 & 0 \\
\frac{\left(k_{3} / 2\right)}{M} & 0 & -\frac{k_{2}+\left(k_{3} / 2\right)}{M} & -\frac{c_{2}}{M} & 0 & -\frac{\alpha_{2}}{M} \\
0 & \frac{\alpha_{1}}{C_{p 1}} & 0 & 0 & -\frac{1}{R_{1} C_{p 1}} & 0 \\
z_{3} \\
z_{2} \\
z_{3} \\
z_{5} \\
z_{6}
\end{array}\right]\left[\begin{array}{c}
z_{1} \\
\\
0 \\
0
\end{array}\right.
$$

Visibly, it is a typical nonlinear, nonsmooth, and discontinuous friction system, which brings some difficulties in determining the moment that the motion of the slider transits from one stage to another stage. The flow chart of the numerical computation is shown in Figure 4.
4.2. Transient Dynamic Analysis Results. The critical friction coefficient value for the generation of self-excited vibration is determined in the above section, which is equal to 0.85 . In this section, friction coefficient is set as $0.6,0.85$, and 1.2, respectively, and used in the transient dynamic analysis. 


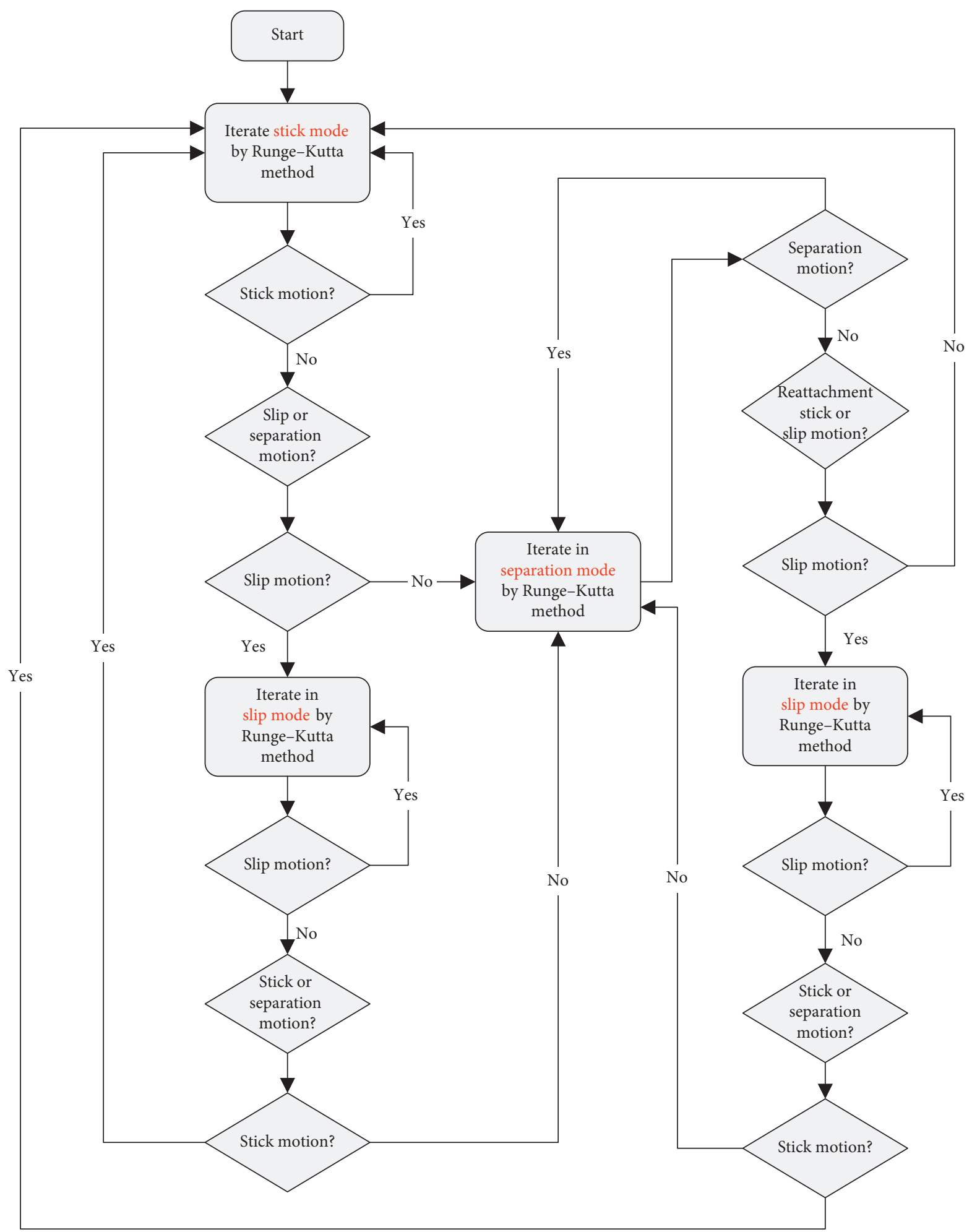

FIGURE 4: The flow chart of the transient dynamic analysis.

These friction coefficients are lower, equal to, and larger than the critical friction coefficient, respectively. The belt velocity $\left(v_{0}\right)$ is set as $0.1 \mathrm{~m} / \mathrm{s}$. The corresponding dynamical behaviors and output voltage are shown in Figure 5. No visible sustained vibration signals can be seen for the friction system at a lower friction coefficient; hence no visible output voltages can be detected. With the friction coefficient increasing to the critical value, strong self-excited vibration appears and consequently visible voltage output is found to be generated in both directions. This phenomenon indicates that the FIV energy converts to electric energy when the FIV appears. In the case of friction coefficient equal to 1.2, the system 


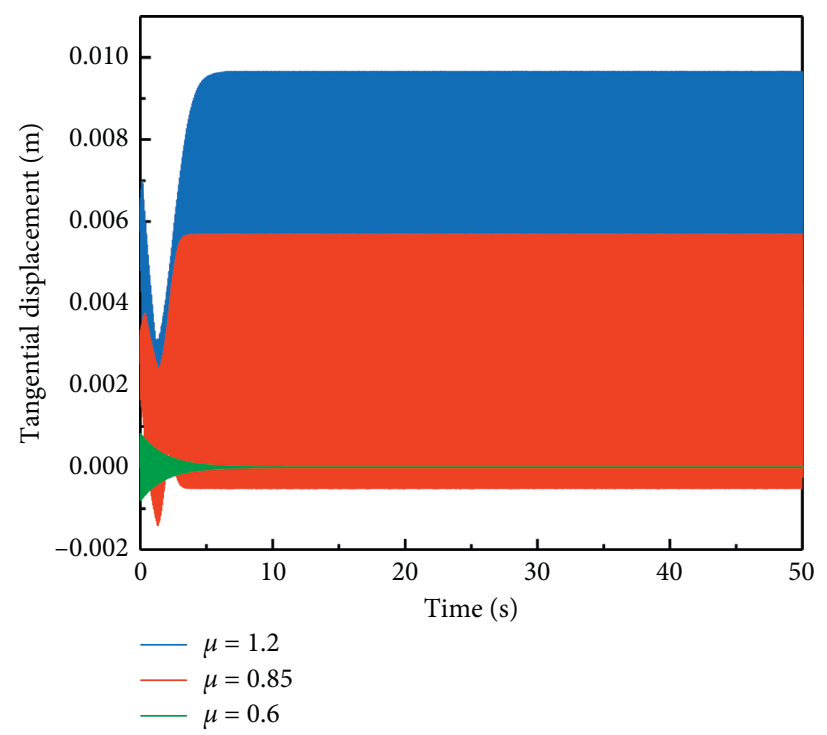

(a)

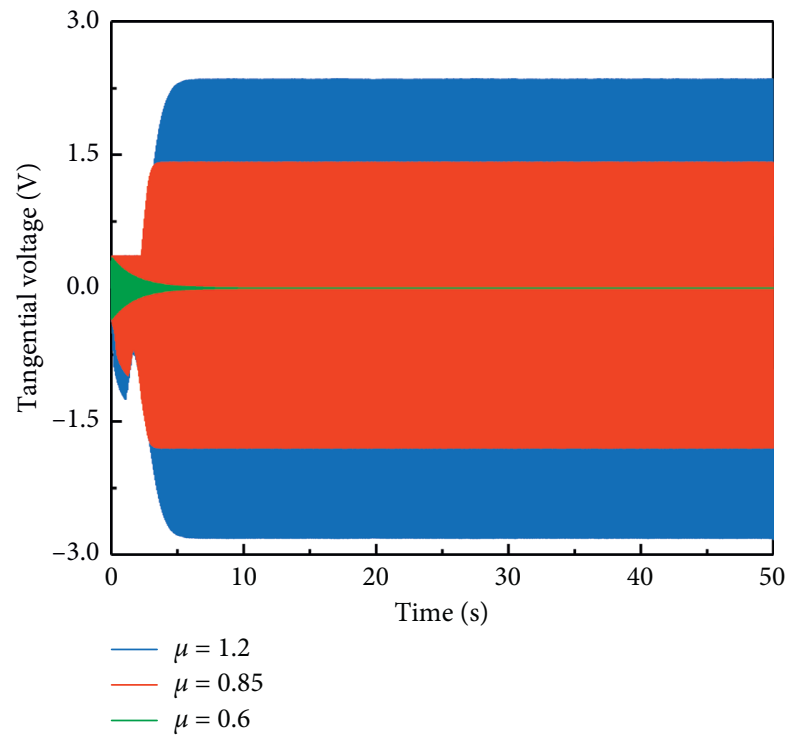

(c)

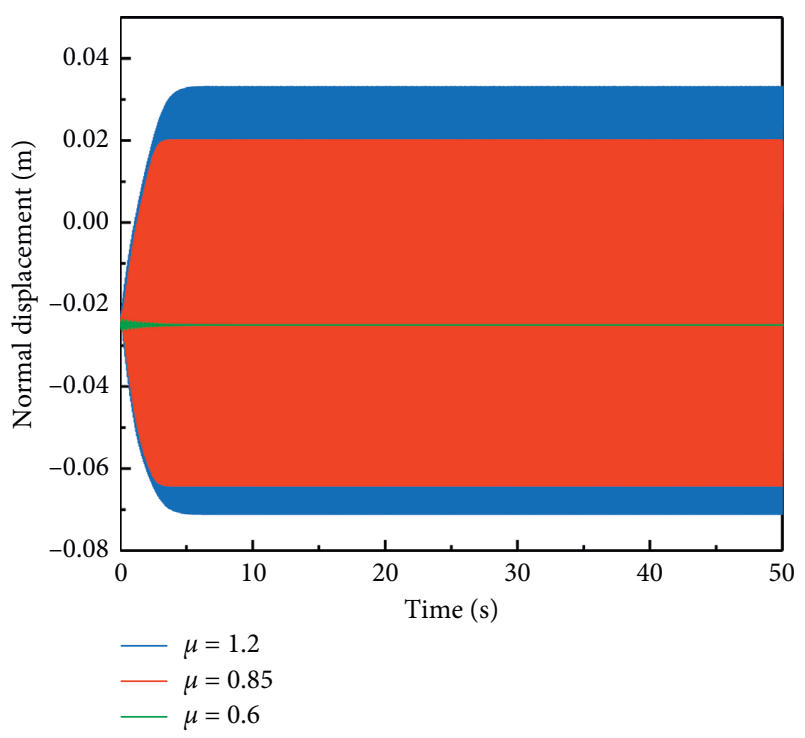

(b)

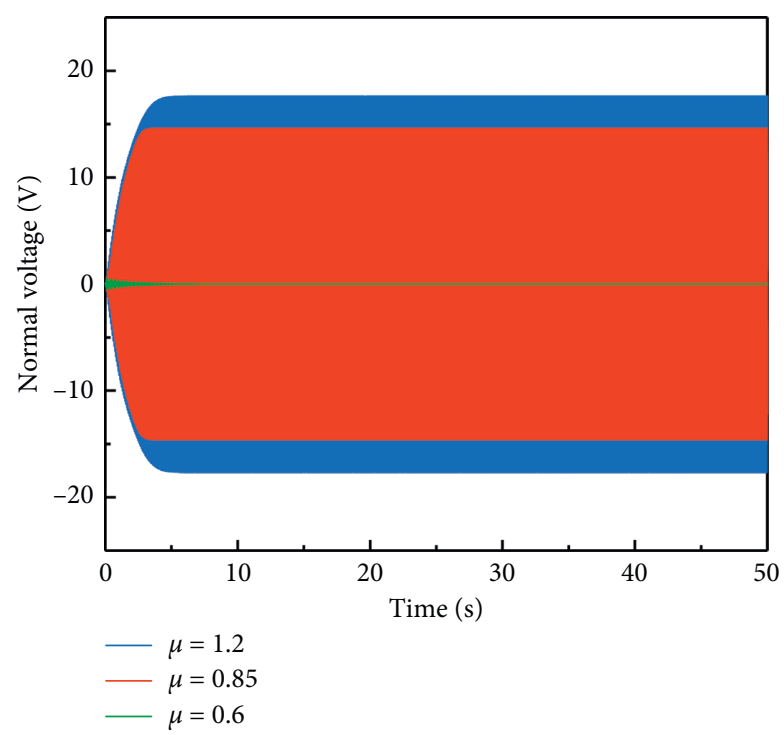

(d)

FIGURE 5: The tangential displacement (a), normal displacement (b), tangential output voltage (c), and normal output voltage (d) of the friction system with different friction coefficients.

exhibits larger amplitude of vibration. As a consequence, higher voltages are outputted in both directions. In addition, it can be seen that the vibration in the normal direction is larger than that in the tangential direction, which accordingly results in a higher output voltage in the normal direction. This is because the normal contact stiffness will not work on the system during the separation stage, which results in the larger displacement in the normal direction, while the friction force disappears and will not act on the slider. Thus, the transient dynamic analysis results further prove the results exhibited in the stability analysis and confirm the flexibility of the PEH by means of FIV.

Figure 6 shows the displacement versus velocity trajectories of friction system with different coefficients in tangential and normal directions, respectively. As can be seen from the periodic orbits appearing in this figure, the steady-state vibration amplitude of the friction system with larger friction coefficient is much larger than that of the friction system with lower friction coefficient, which further indicates that larger friction coefficient leads to a stronger vibration level. Additionally, due to the consideration of separation and reattachment behavior between the slider and belt, the slider's tangential stick-slip motion becomes unconventional: sometimes the speed of the slider can be even larger than the belt speed during the friction process and forms an interesting stick-slip limit cycle.

Expectedly, the large-amplitude periodic (limit-cycle) response on the high-energy orbit of the friction system with larger coefficient is also observed in the velocity versus voltage trajectory shown in Figure 7. For a larger 


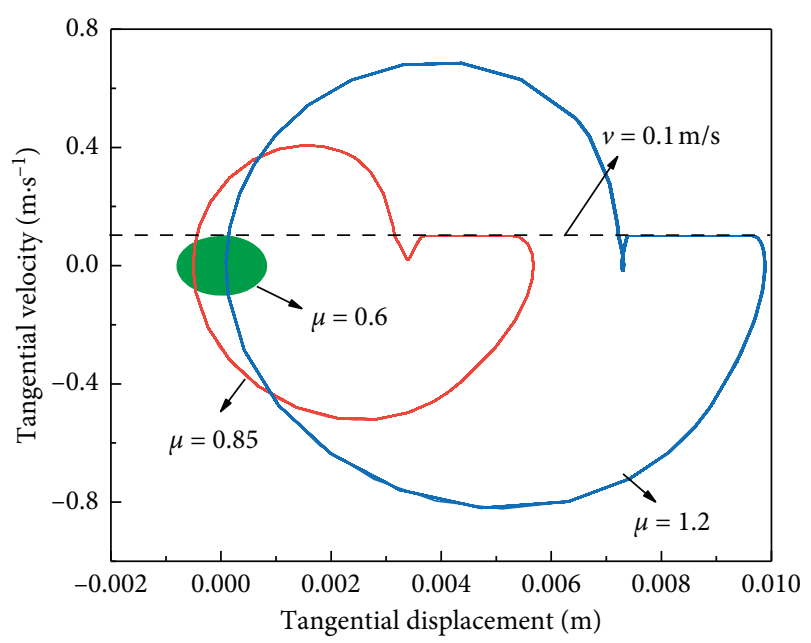

(a)

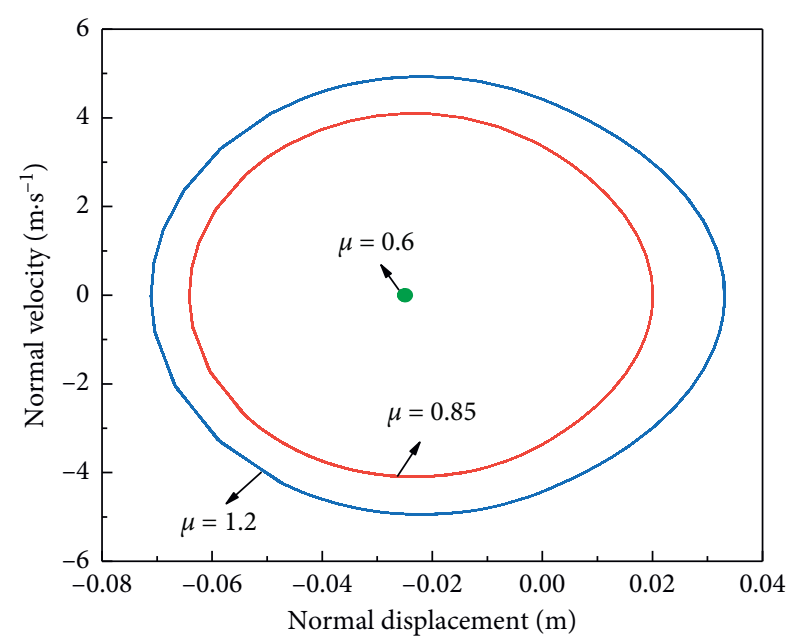

(b)

Figure 6: The tangential displacement versus tangential velocity. (a) Normal displacement versus normal velocity. (b) Phase portraits of the friction system.

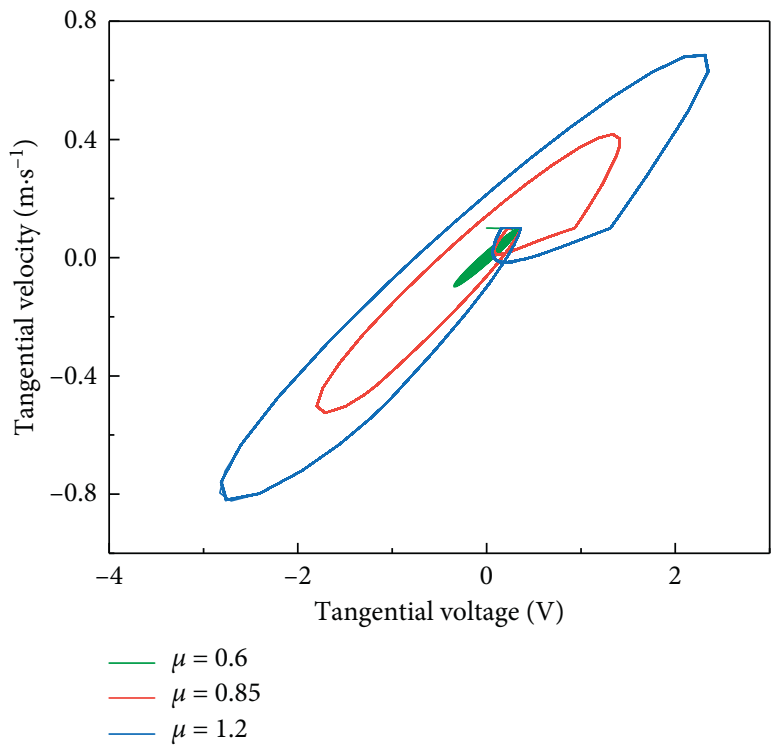

(a)

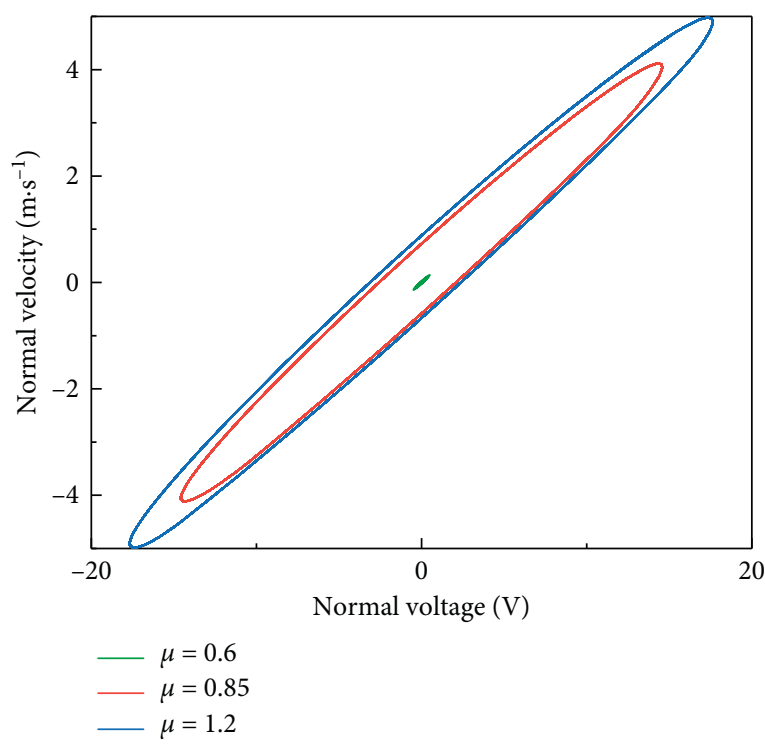

(b)

FIGURE 7: The open-circuit voltage versus velocity phase trajectories.

friction coefficient, the orbit exhibits a large-amplitude limit cycle, which indicates that a sustained and stable voltage can be outputted during the friction process, while for the friction system with lower friction coefficient, the orbit gradually evolves to a point with the increase of time, suggesting that no visible FIV energy is transferred to electric energy. This phenomenon further verifies the role of friction coefficient in affecting the stability and energy harvesting behaviors of friction system and also supports the opinion that the FIV can be transferred to electric energy by piezo elements.

\section{Impact Factors Analysis}

It has been reported that the dynamics of friction system are very sensitive to the contact parameters [38-40], which indicates that the variation of working parameters can significantly change the energy harvesting behaviors of friction system as well. In this section, the effect of friction coefficient on the dynamics and energy harvesting is studied. Then, the dynamics and energy harvesting behaviors of the friction system with and without consideration of the separation between slider and belt are analyzed, respectively. 
Finally, the dynamics behaviors of the friction system with different external electric resistances are analyzed, and the role of electric resistances in affecting $\mathrm{PEH}$ performance is revealed.

5.1. Friction Coefficient. According to the value of critical friction coefficient (0.85), the dynamic behaviors of the friction system with kinetic and static friction coefficients, denoted by $\mu_{k}$ and $\mu_{s}$, located within three ranges $\left(\mu_{k}<\mu_{s}<\mu_{c}, \mu_{k}<\mu_{c}<\mu_{s}\right.$, and $\left.\mu_{c}<\mu_{k}<\mu_{s}\right)$, are examined and compared. The values of friction coefficients in these three cases are listed in Table 2, and concurrently listed in Table 2 are the Eps of the friction system under different friction coefficients. The initial condition is set at $\left(x_{\mathrm{e}}, 0.1, y_{\mathrm{e}}, 0,0,0\right)$, with the tangential velocity of the slider equaling the velocity of the belt. The external electric resistance in this analysis is set at $10 \mathrm{~K} \Omega$.

Figure 8 depicts the dynamical behaviors and output voltages of the friction system with three different cases of friction coefficients. Visibly, the vibration level and output voltage are strongly related to the friction coefficient. In the tangential direction, the friction system exhibits the strongest self-excited vibration level when the friction coefficient is located in Case 3. Accordingly, the voltage generated in this situation exhibits the highest value. Meanwhile, for the lowest friction coefficient of Case 1, the tangential vibration amplitude and output voltage are visibly lower than those generated from the other two cases (Figures 8(a) and 8(c)). Similarly, for the normal direction, a larger coefficient is found to be able to generate stronger vibration and higher output voltage as well. Therefore, the vibration level and voltage values generated in Case 3 are larger than those from Case 2, and vibration and voltage signals generated from Case 1 show the lowest values.

Figure 9 shows the phase plots of friction system with different friction coefficient cases. For Case 1, the tangential stick-slip limit cycle is very complicated and exhibits lots of different cycles of stick-slip motion. Similarly, the normal vibration also shows a multiperiod limit cycle. Meanwhile, for Case 2, the phase trajectory in tangential direction is very unconventional, in which several limit cycles are characterized by appearing and repeating themselves; additionally, the tangential velocity of slider exceeds the belt velocity $(v>0.1 \mathrm{~m} / \mathrm{s})$ and causes overshoot phenomenon during the vibration. Additionally, the normal vibration also forms a multiperiod limit cycle. Case 3 represents the situation in which both the static and kinetic friction coefficients are larger than the critical friction coefficient; it is seen that the tangential stick-slip and overshoot always happen. In this case, the friction system has a large-amplitude (limit-cycle) response in both the normal direction and the tangential direction, compared with the other two cases.

The velocity versus voltage trajectory of the friction system in three cases of friction coefficient is plotted. In the tangential direction, it is visible that the orbit of friction system with Case 3 possesses biggest limit cycle, which suggests that the friction system can generate higher amplitude voltage than the other two cases. Meanwhile the
TABLE 2: The values of friction coefficients and the corresponding Eps in these three cases.

\begin{tabular}{lcccc}
\hline Case & $\mu_{k}$ & $\mu_{s}$ & $\mu_{c}$ & Eps $\left(x_{\mathrm{e}}, y_{\mathrm{e}}\right)$ \\
\hline 1 & 0.4 & 0.6 & 0.85 & $(-0.00216,-0.0258)$ \\
2 & 0.7 & 0.9 & 0.85 & $(0.00104,-0.0246)$ \\
3 & 1 & 1.2 & 0.85 & $(0.00397,-0.0235)$ \\
\hline
\end{tabular}

output voltage amplitude in Case 1 shows the smallest limit cycle. Indeed, both trajectories from Case 1 and Case 2 exhibit very complicated orbits. In the normal direction, as expected, the orbit of friction system with Case 3 exhibits the largest amplitude of limit cycle, which indicates that a sustained and high voltage is outputted in this direction during the sliding process. Additionally, the orbit of friction system with Case 1 forms a smallest limit cycle, which reflects that relative lower voltage is generated in this situation. This phenomenon can well reflect the time domain signal of voltage shown in Figure 8.

To sum up, the dynamic and output voltages are strongly related to the relationship among the kinetic friction coefficient $\mu_{k}$, static friction coefficient $\mu_{s}$, and critical friction coefficient $\mu_{c}$. The friction system can generate stronger vibration and output higher voltage when the friction coefficient is located in the range of $\mu_{c}<\mu_{k}<\mu_{s}$. In contrast, lower vibration and less voltage are generated when the friction coefficient locates in the range of $\mu_{k}<\mu_{s}<\mu_{c}$.

\subsection{Comparison and Analysis of Ignoring/considering} Separation. In most previous studies of FIV of small models, it is assumed that the slider is still in contact with belt during vibration process $[50,60]$. Although the possibility of loss of contact was mentioned in $[61,62]$, the effect of separation was not studied. As the unstable FIV caused by the friction force at the contact interface grows, it is important to consider separation during the vibration, when friction temporarily disappears. The loss of contact will affect not only the contact behaviors of friction system but also the energy harvesting behaviors. Therefore, in this section, the dynamics and energy harvesting behaviors of the friction system in both cases, that is, ignoring separation and considering separation between slider and the belt, are analyzed and compared.

The flow chart of transient dynamic analysis when the friction system ignores the separation between slider and belt is shown in Figure 10. Due to ignoring the separation, the equations of motion are given by equation (2). The iteration process starts from the stick mode, and slider velocity is checked at the end of every single time step during numerical computation. Once the slider velocity is not equal to the belt velocity $(0.1 \mathrm{~m} / \mathrm{s})$, the iteration process switches to the slide mode, and initial value of slide mode is the last value of the friction system in the stick mode. Then, the slider rubs on the moving belt until the next stick happens.

In the previous study, it is found that the separation can occur in Case 2 and Case 3; thus, it is worth noting that comparison between ignoring separation and considering separation will be performed in these two cases. 


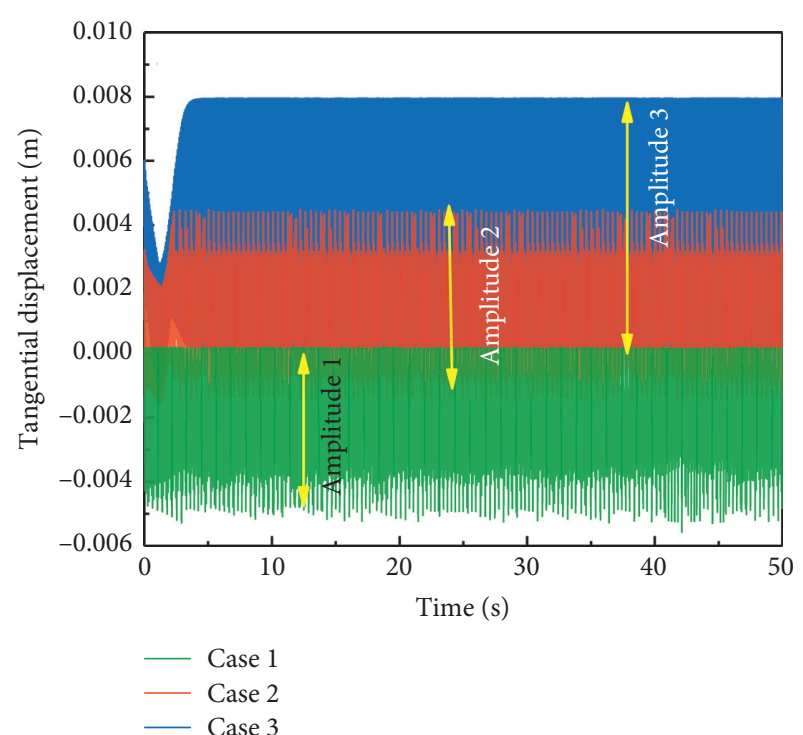

(a)

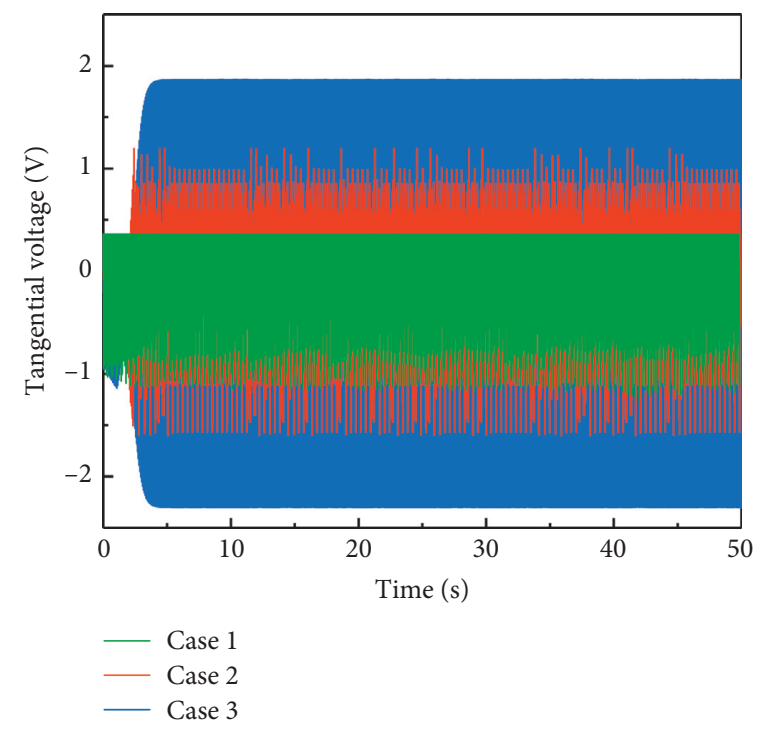

(c)

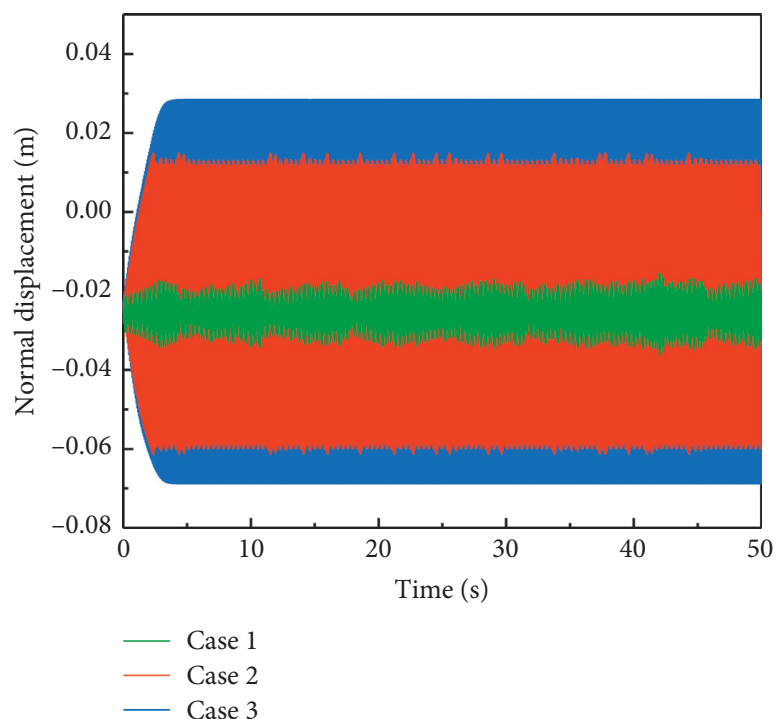

(b)

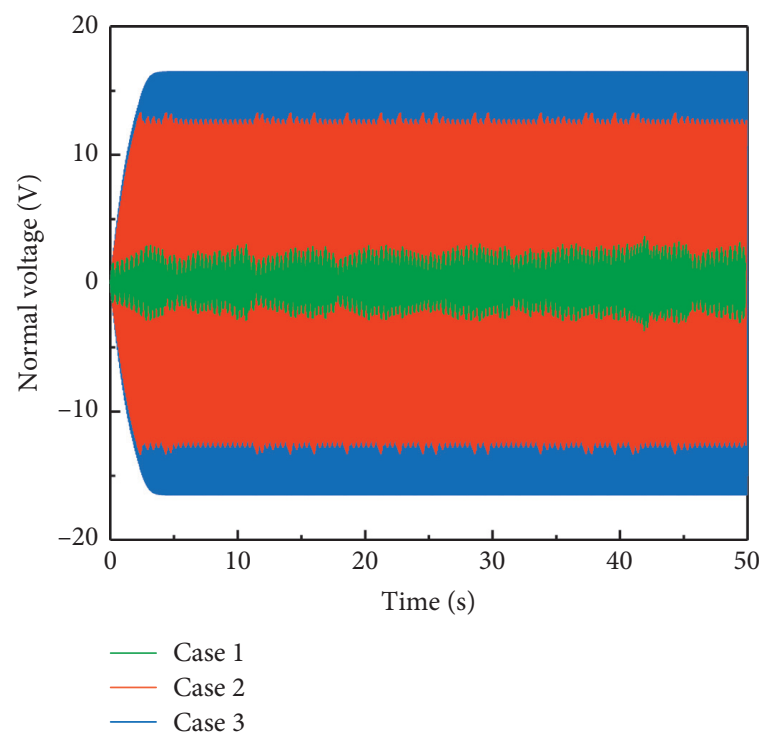

(d)

Figure 8: The tangential displacement (a), normal displacement (b), tangential output voltage (c), and normal output voltage (d) of the friction system with three different cases of friction coefficients.

5.2.1. $\mu_{k}<\mu_{c}<\mu_{s}$. Numerical results with $\mu_{k}=0.7, \mu_{\mathrm{s}}=0.9$, and initial condition $\left(x_{\mathrm{e}}, 0.1, y_{\mathrm{e}}, 0,0,0\right)$ are shown in Figure 11 . Visibly, the separation and reattachment behaviors make both the tangential and normal dynamics of friction system different from their counterparts when separation is ignored. For the tangential direction, the displacement mainly reflects in the positive direction when considering separation, while when separation is ignored, the displacement fluctuates between the positive and negative directions, suggesting that the slider moves back and forth with the moving belt. In addition, the amplitude of displacement fluctuation in the case of considering separation is larger than that in the case of ignoring separation, as shown in Figure 11(a); thus the output voltage in this direction is larger in the case of considering separation (Figure 11(c)). Meanwhile, for the normal direction, the situation changes significantly. It can be seen that the normal displacement amplitude when considering separation is smaller compared with the situation of ignoring separation; accordingly the output voltage is smaller when separation is taken into consideration, as shown in Figures 11(b) and 11(d).

The contact forces of these two situations are plotted in Figure 11(e). For the case when separation is considered, multiple separation events can be observed as the values of contact force vary from zero to positive and then drop to zero during the vibration. Meanwhile, when ignoring separation, the value of contact force gradually grows and 


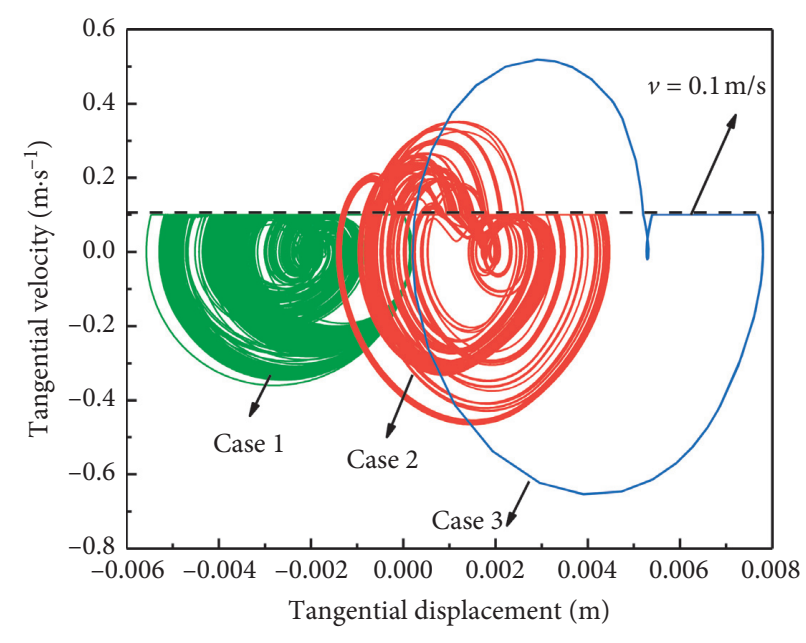

(a)

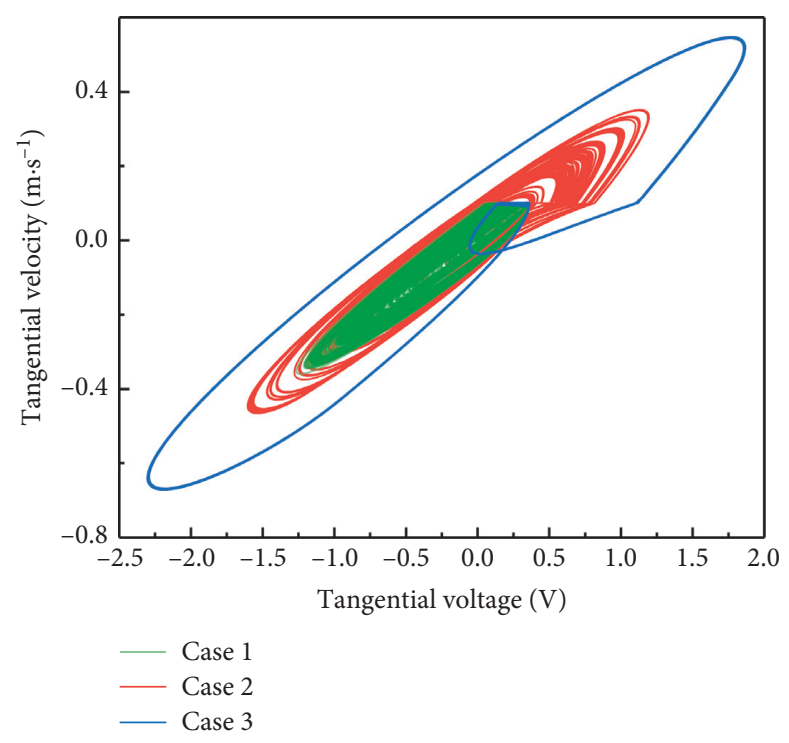

(c)

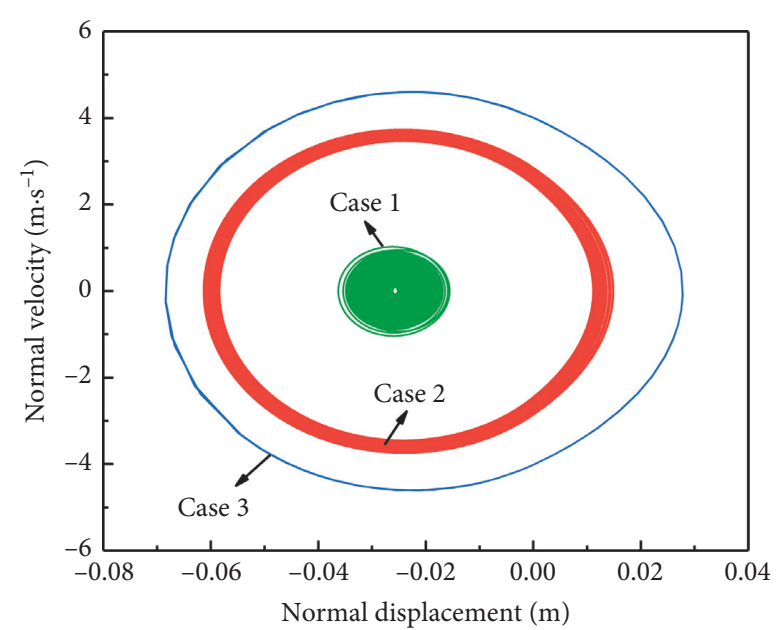

(b)

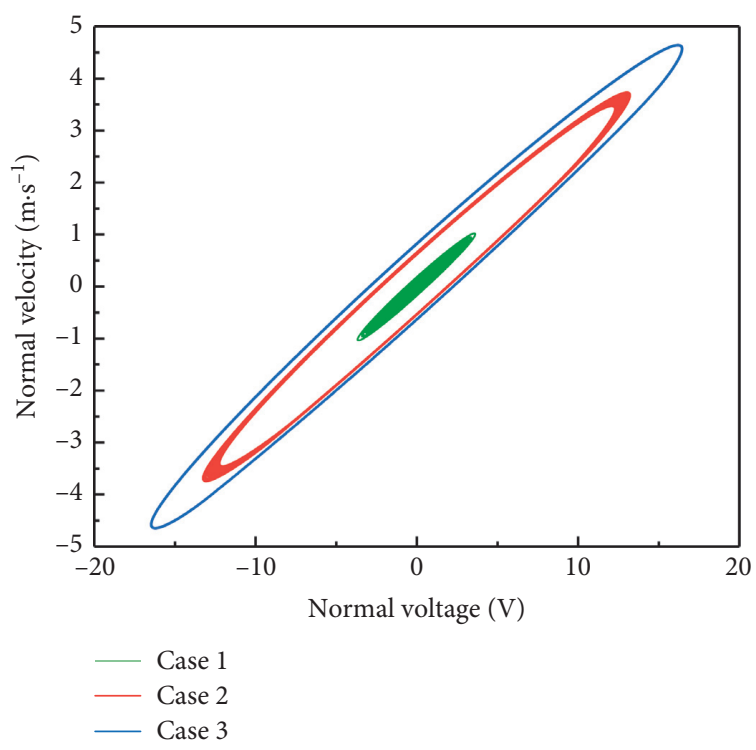

(d)

Figure 9: The displacement verus velocity trajectory of the friction system in tangential direction (a) and normal direction (b). The velocity versus voltage trajectory of the friction system in tangential direction (c) and normal direction (d).

becomes negative, and the amplitude is larger than that in the case of considering separation. This phenomenon well reflects the results shown in the displacement and output voltage signals.

The displacement verus velocity phase trajectories of the friction system in both directions are shown in Figures 12(a) and 12(b). For the case of ignoring separation, the tangential phase trajectory exhibits a typical stick-slip limit cycle, while the phase trajectory in the case of considering separation is very unconventional, in which several limit cycles appear and repeat themselves; in addition, the slider is found to be able to slide on the belt with its speed passing through the speed of the belt without stick motion. For the normal direction, when separation is ignored, the phase trajectory exhibits a larger-amplitude limit cycle compared to the cases of considering separation.
Furthermore, the velocity versus voltage trajectory is plotted in Figures 12(c) and 12(d). Visibly, the trajectory in the tangential direction when considering separation exhibits a larger-amplitude and more complicated limit cycle, compared to the case of ignoring separation. This is attributed to the fact that the velocity of slider can sometimes exceed the belt velocity. As a consequence, the tangential output voltage when considering separation is visibly larger compared to the situations of ignoring separation (Figure 12(c)). Meanwhile, for the trajectory in the normal direction, for the case of ignoring separation, the trajectory exhibits a larger-amplitude limit cycle, which indicates that a sustained and stable voltage can be outputted during the friction process. In contrast, when separation is taken into consideration, the trajectory shows a smaller limit cycle, suggesting that less voltage can be outputted in this situation (Figure 12(d)). 


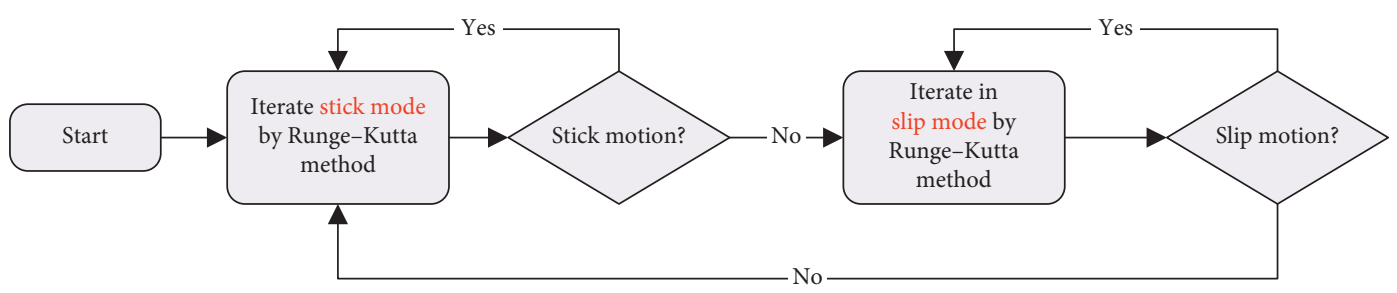

Figure 10: The flow chart of transient dynamic analysis when the friction system ignores the separation between belt and slider.

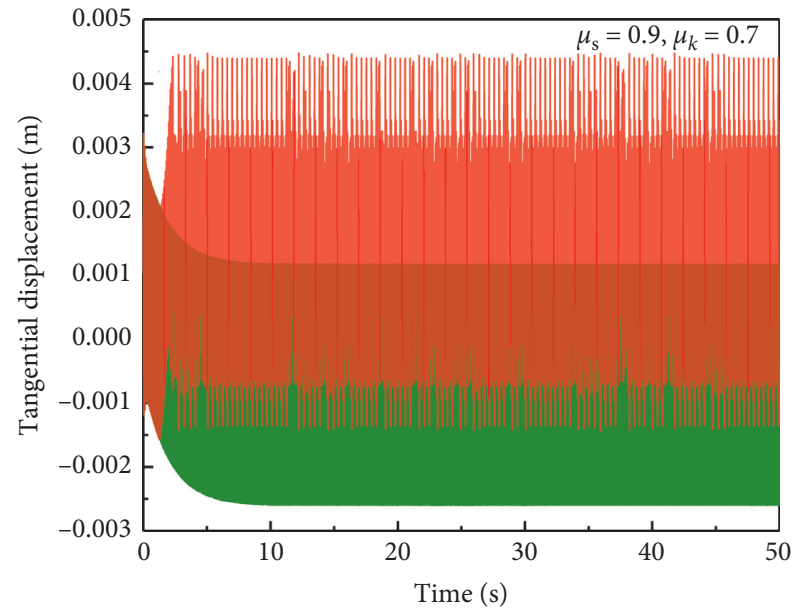

- Ignoring separation

- Considering separation

(a)

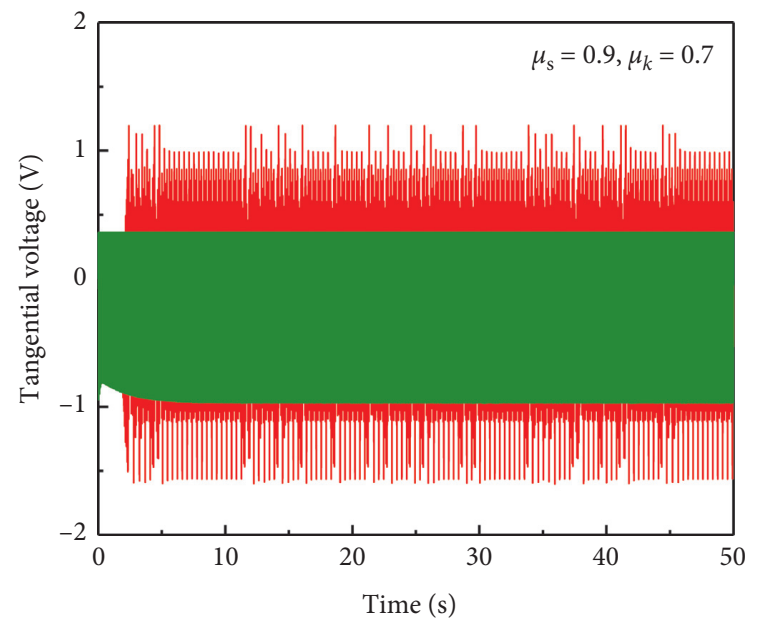

_ Ignoring separation

— Considering separation

(c)

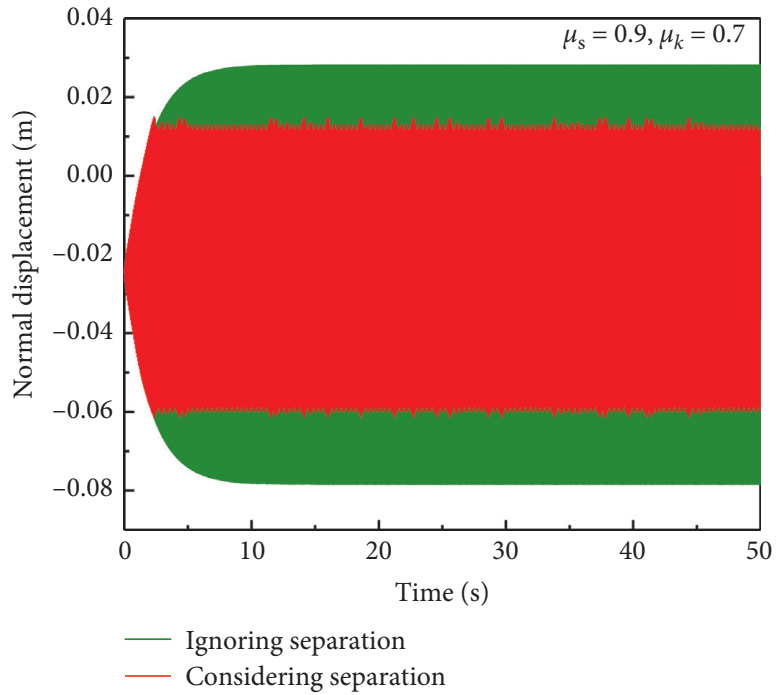

(b)

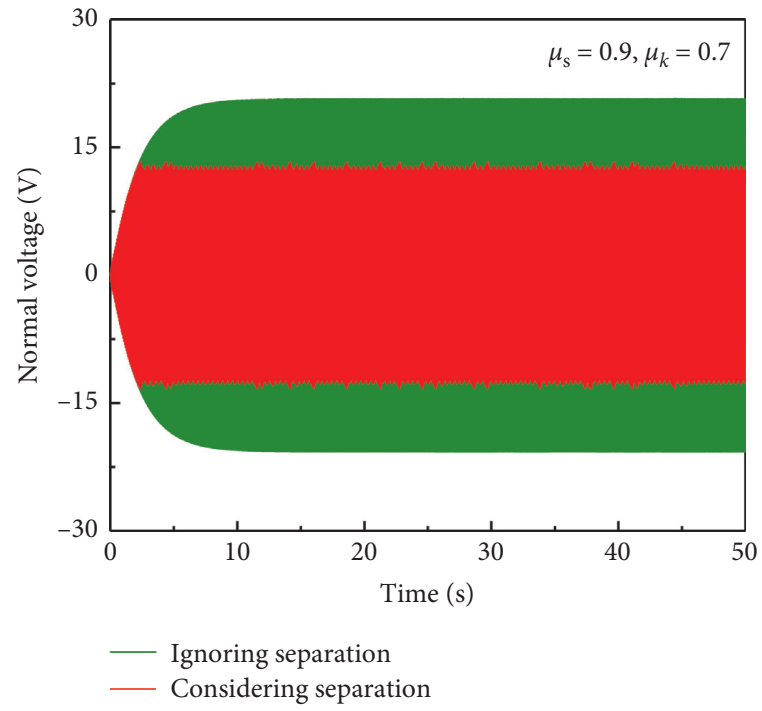

(d)

FIgURE 11: Continued. 


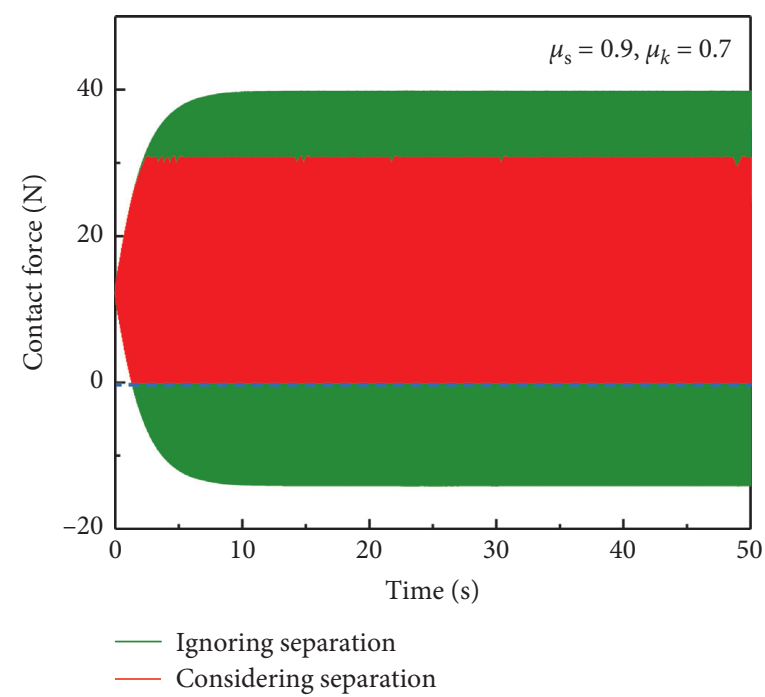

(e)

Figure 11: The tangential displacement (a), normal displacement (b), tangential voltage (c), normal voltage (d), and contact force (e) of the friction system in the time domain when $\mu_{k}=0.7$ and $\mu_{s}=0.9$.
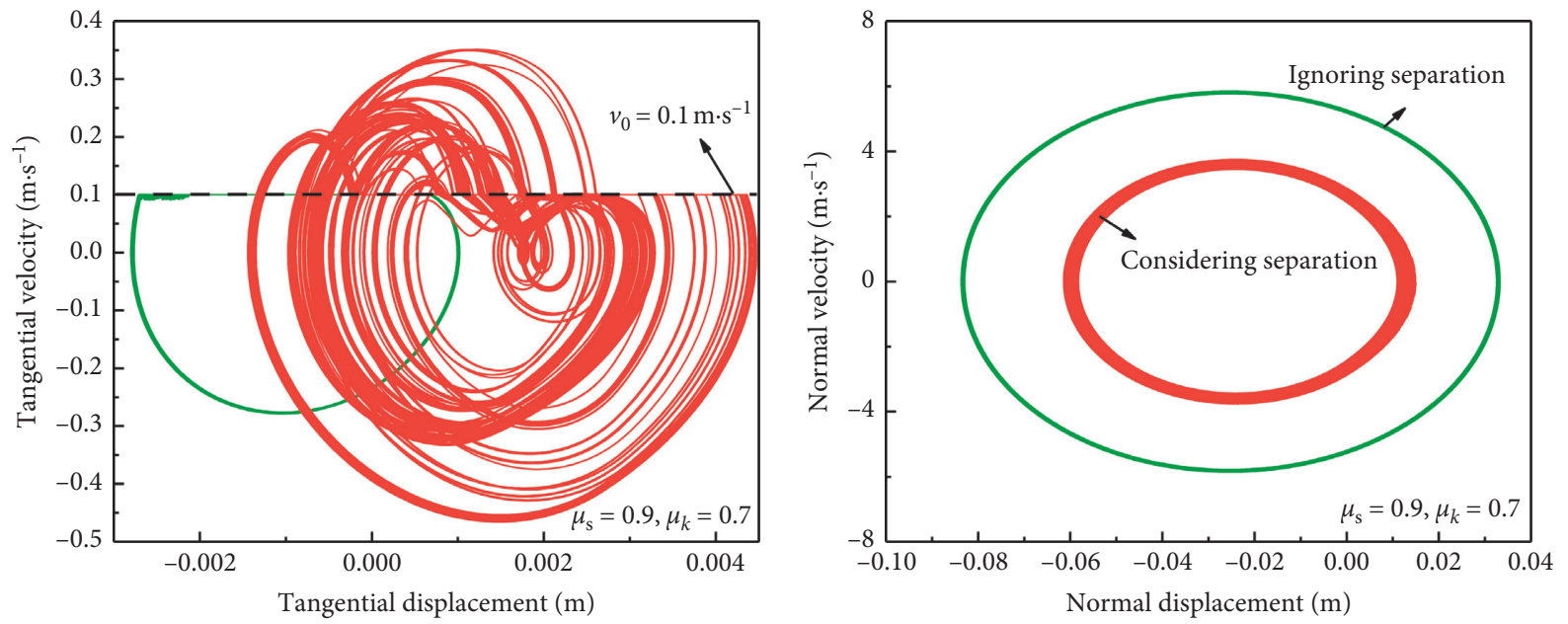

- Ignoring separation

_ Considering separation

(a)

(b)

Figure 12: Continued. 


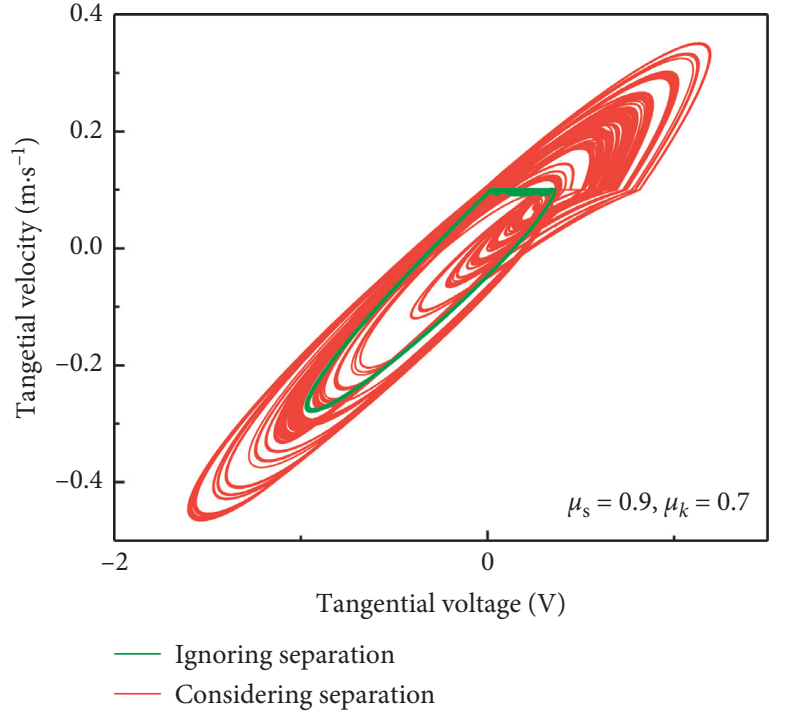

(c)

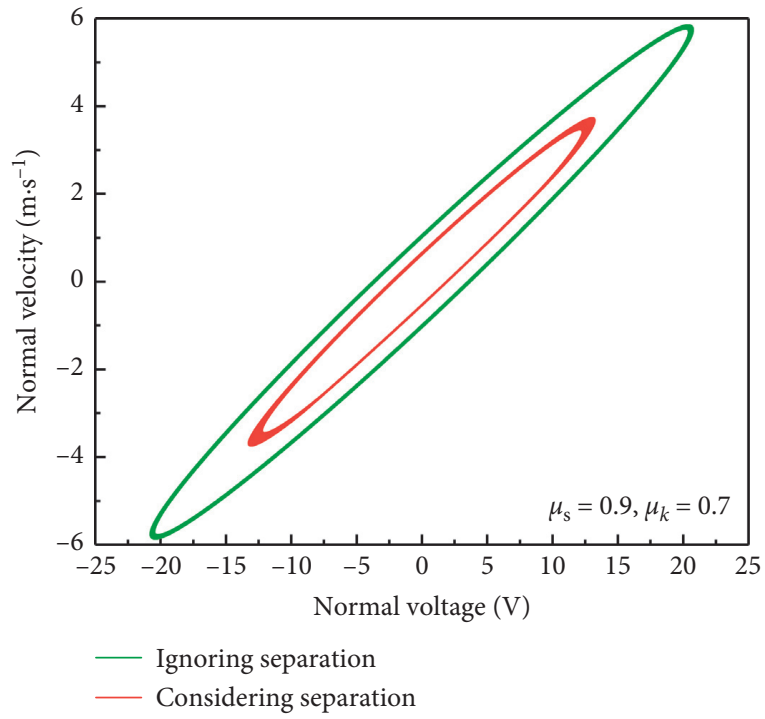

(d)

FIGURE 12: The displacement versus velocity phase trajectory of the friction system in tangential direction (a) and normal direction (b). The velocity versus voltage trajectory of the friction system in tangential direction (c) and normal direction (d) when $\mu_{k}=0.7$ and $\mu_{s}=0.9$.

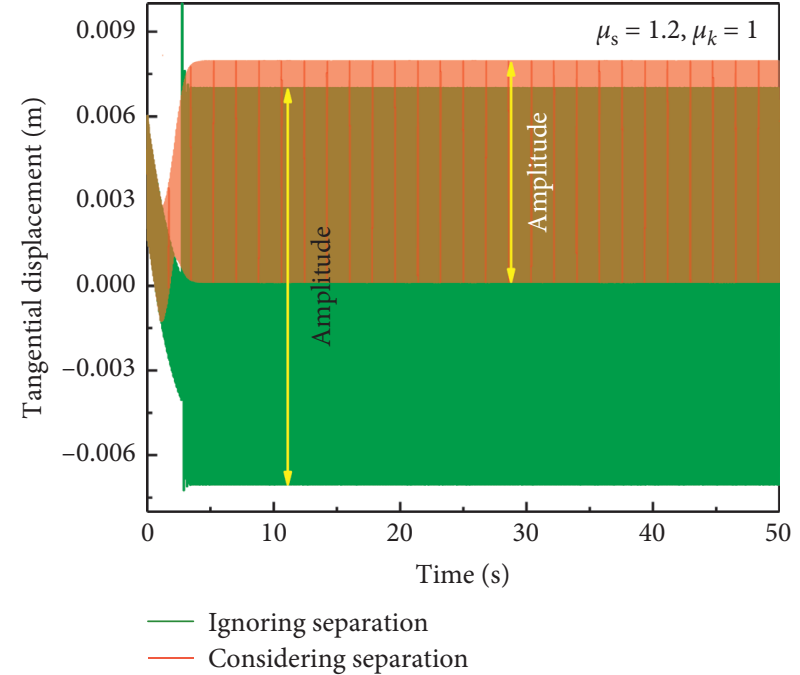

(a)

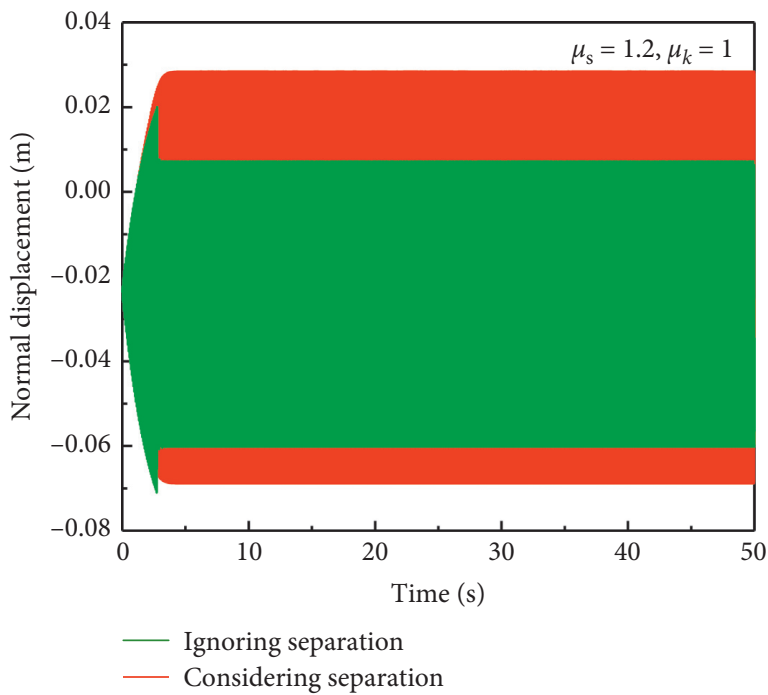

(b)

Figure 13: Continued. 


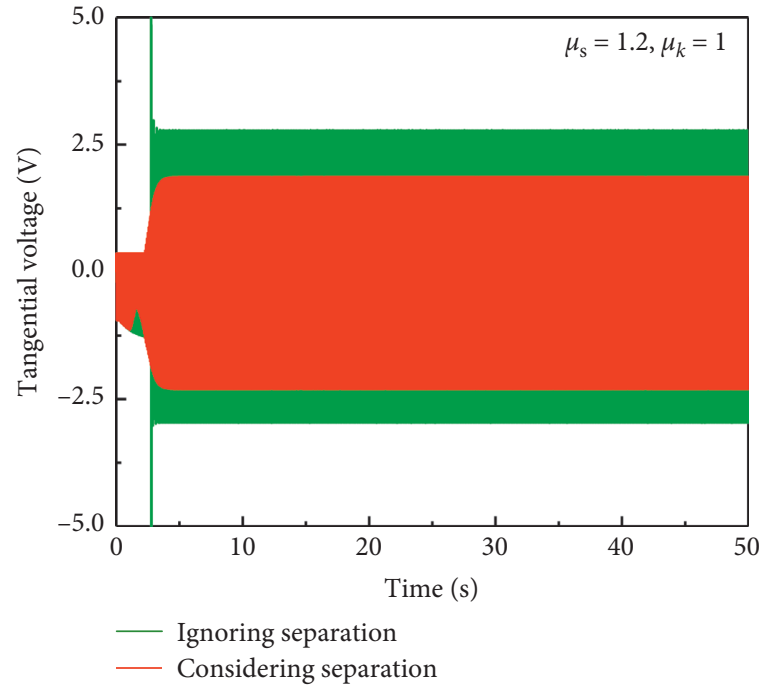

(c)

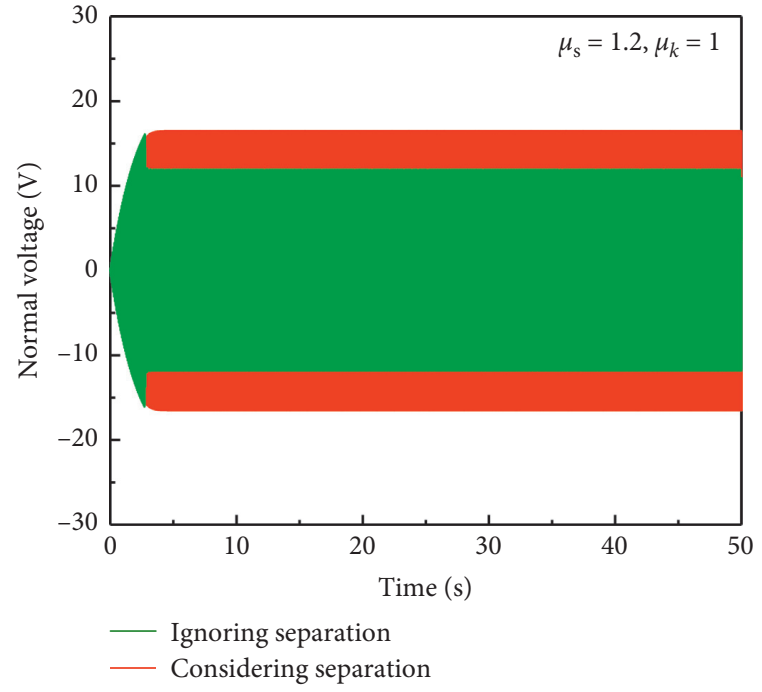

(d)

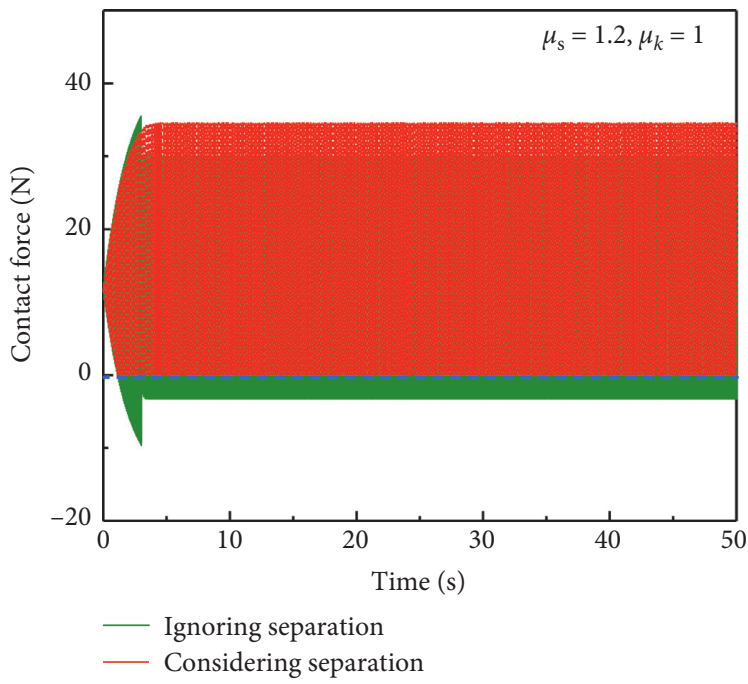

(e)

FiguRE 13: The tangential displacement (a), normal displacement (b), tangential voltage (c), normal voltage (d), and contact force (e) of the friction system in the time domain when $\mu_{k}=1$ and $\mu_{s}=1.2$.

5.2.2. $\mu_{c}<\mu_{k}<\mu_{s}$. Figure 13 shows the simulation results in the case where $\mu_{k}=1$ and $\mu_{\mathrm{s}}=1.2$, and the initial condition of system is set at $\left(x_{\mathrm{e}}, 0.1, y_{\mathrm{e}}, 0,0,0\right)$. It can be seen that the dynamics and output voltage behaviors show significant difference between these two cases (ignoring and considering separation). For the tangential direction, the displacement signal shows larger amplitude compared with the situation of considering separation. However, the displacement amplitude in the normal direction shows totally opposite results (Figure 13(a) and 13(b)). Consequently, the output voltage in tangential direction when ignoring separation is visibly larger than that in the case of considering separation, while the normal output voltage is smaller compared with the situation of considering separation (Figure 13(c) and 13(d)). This phenomenon is also reflected in the signals of contact force in these two cases (ignoring and considering separation); it can be seen that the contact force in the case of considering separation is larger than that in the case of ignoring separation (Figure 13(e)).

Expectedly, when separation is ignored, the displacement versus velocity trajectory shows larger limit cycle compared to the case of considering separation in the tangential direction, and thus the corresponding velocity versus voltage trajectory when ignoring separation possesses larger limit cycle in this direction, as shown in Figures 14(a) and 14(b). This phenomenon suggests that ignoring separation will overestimate vibration level and energy harvesting ability in the tangential direction of friction system. Conversely, when ignoring separation, the displacement versus velocity limit cycle in the normal direction exhibits much smaller amplitude than the case of considering separation, and the corresponding velocity versus voltage trajectory 


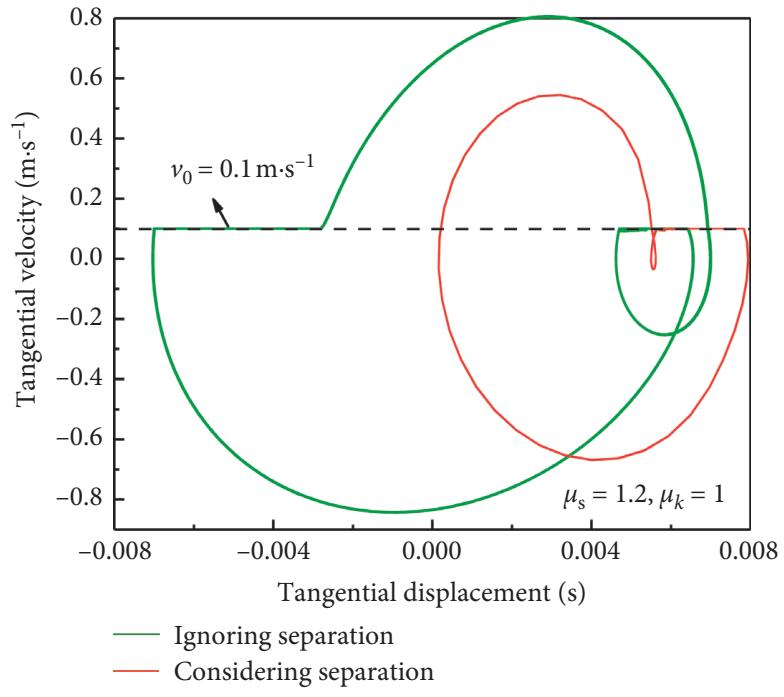

(a)

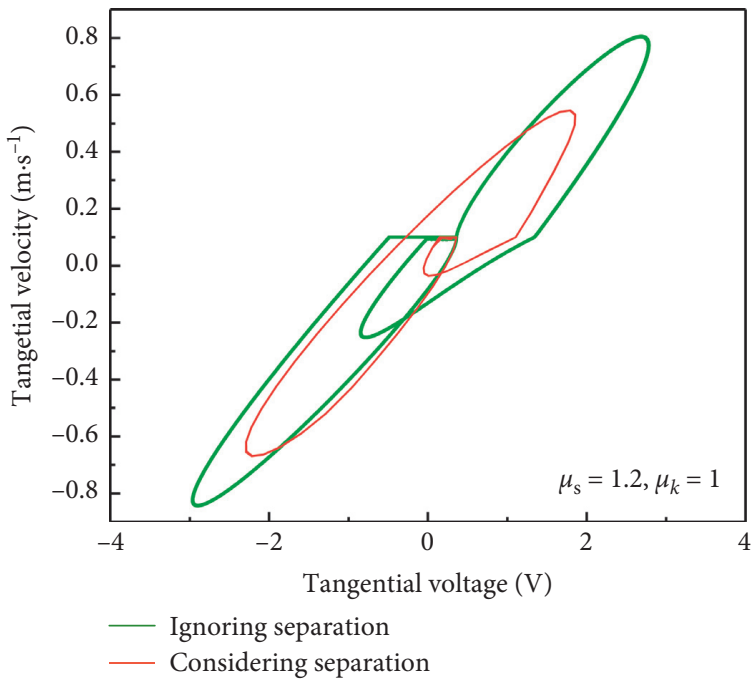

(c)

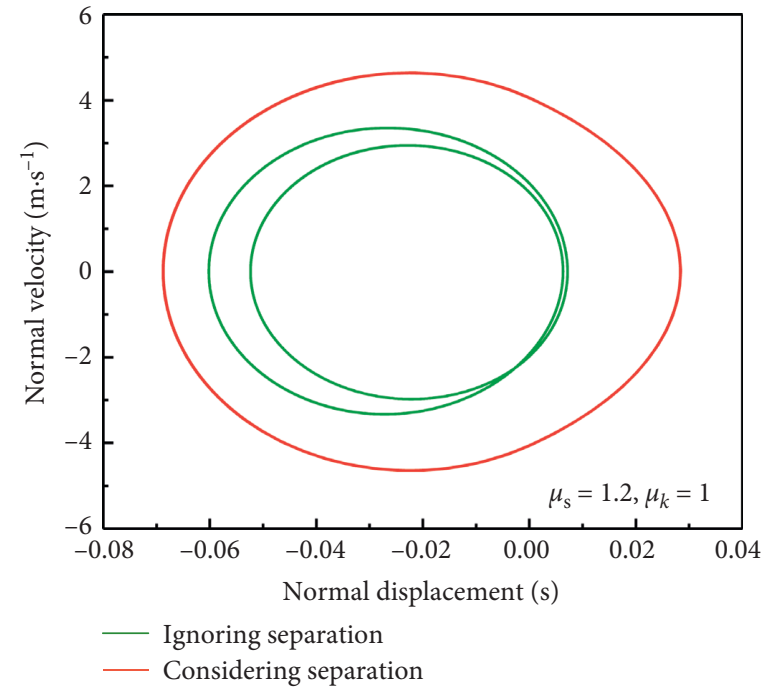

(b)

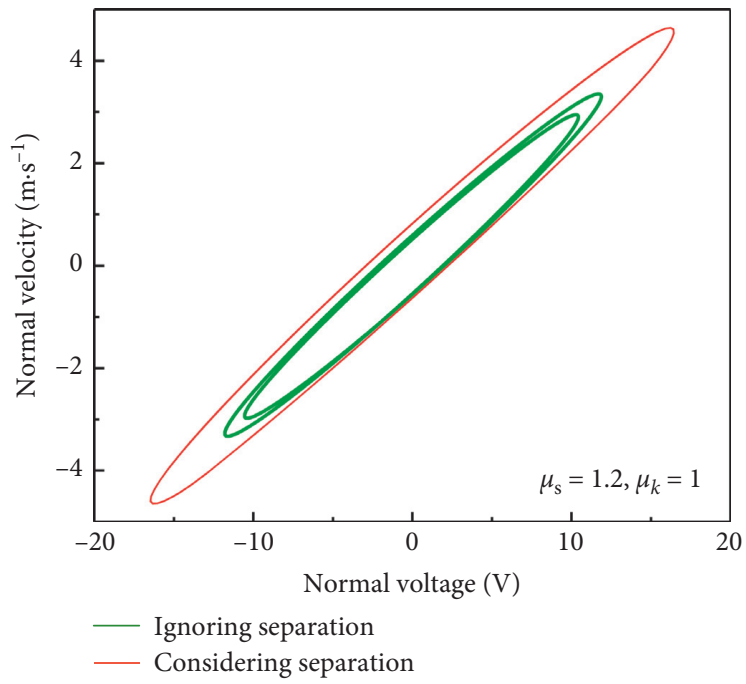

(d)

FiguRE 14: The displacement versus velocity phase trajectory of the friction system in tangential direction (a) and normal direction (b). The velocity versus voltage trajectory of the friction system in tangential direction (c) and normal direction (d) when $\mu_{k}=1$ and $\mu_{s}=1.2$.

possesses smaller limit cycle in this direction, which indicates that ignoring separation will underestimate the vibration level and energy harvesting ability in the normal direction of friction system, as shown in Figures 14(c) and 14(d).

Combining the puzzling results shown in these two situations, a possible physical explanation is provided. When ignoring the separation behavior of the friction interface, the normal spring is stretched and provides continuous resistance to the slider, which accordingly prevents the slider from continuously moving upward, while the friction force still contributes to the system vibration in the tangential direction. In contrast, when separation is considered in this system, the normal contact stiffness will lose its effect when separation occurs; at this time, the friction force disappears, and the normal contact stiffness will not provide resistance force to the normal vibration. Since the tangential and normal vibration is coupled, the vibration of this system when separation is considered is actually more complex. The friction coefficient also plays a significant role in affecting the dynamics and energy harvesting performance of the friction system; visibly, the different friction coefficient will cause complete opposite results. These are very interesting findings and they are useful for designing PEH devices via FIV.

5.3. Comparison and Analysis of the Friction System with Different Electric Resistances (R). In this section, the effect of $R$ on the dynamic and output voltage is studied. Figure 15 exhibits the dynamical behaviors and output voltages of the friction system under different values of $R$. It is found that the variation of $R$ will not cause vibration level of friction system generating significant variation. However, $R$ is found to be able to affect the output voltages values in both 
$R=10 \mathrm{~K} \Omega$

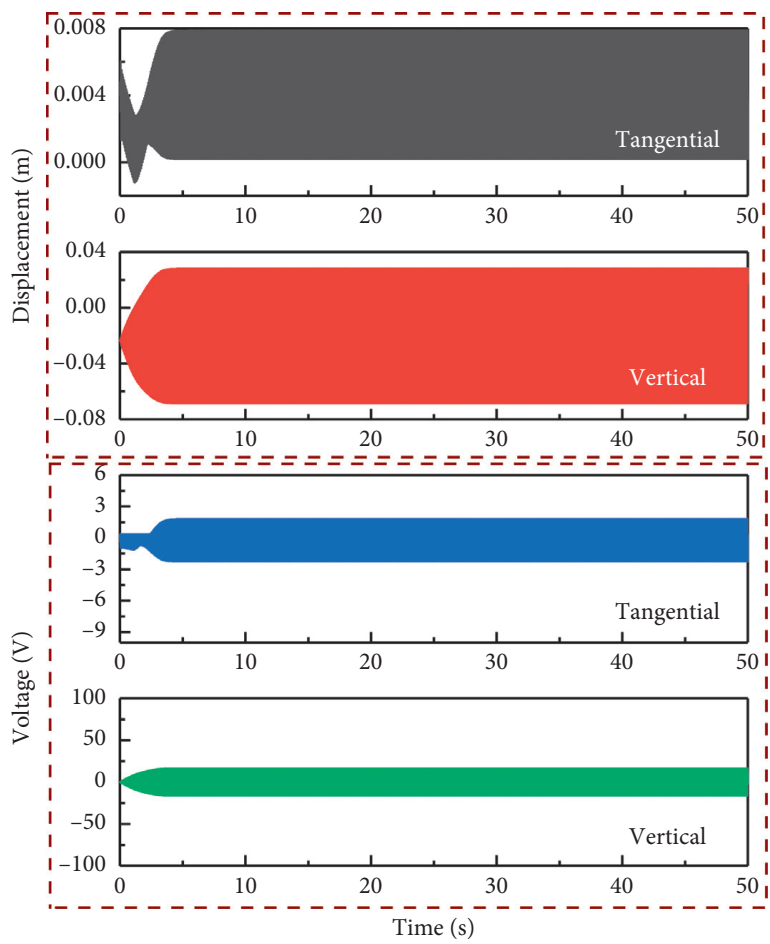

(a)

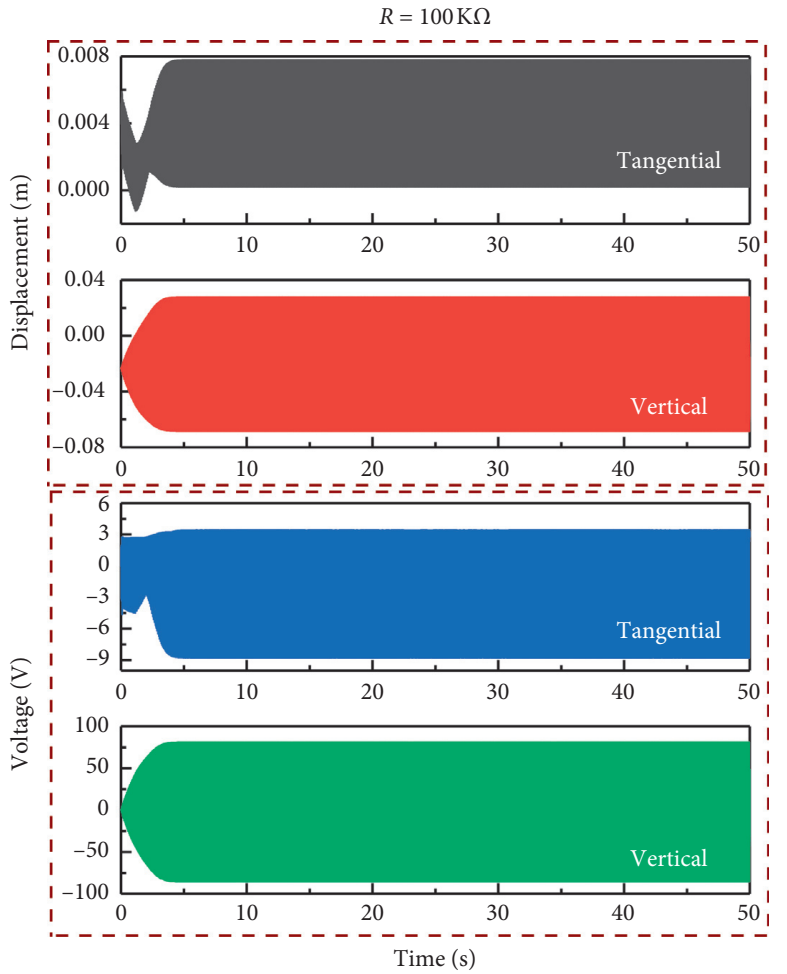

(c)

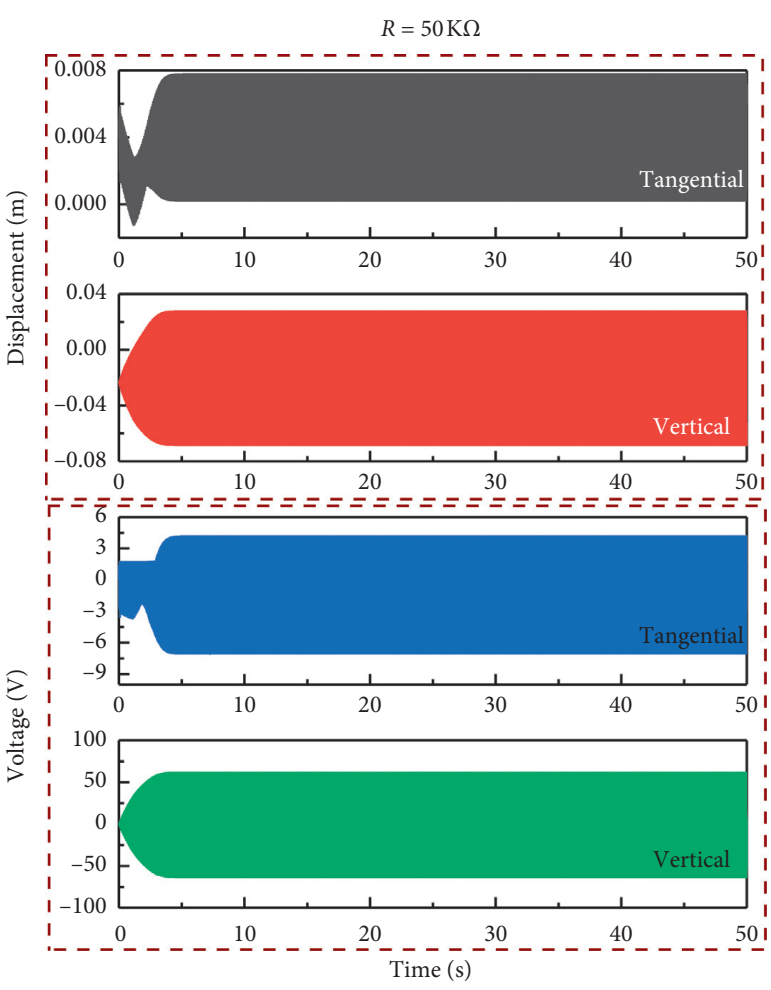

(b)

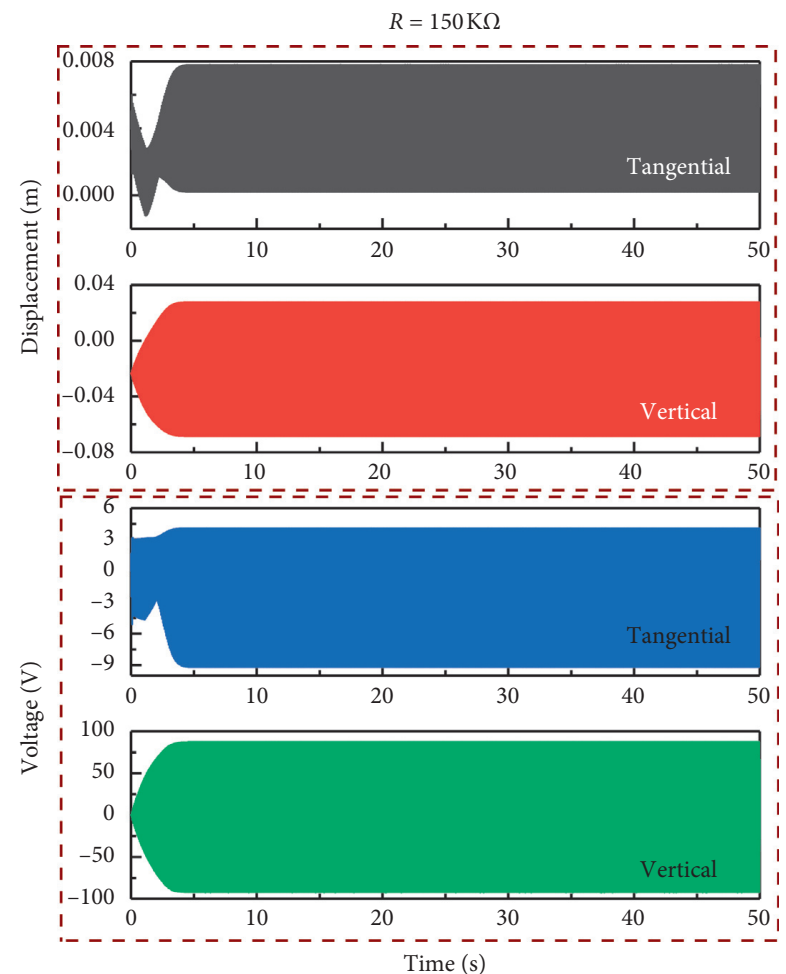

(d)

FIgURE 15: The displacement and output voltage of friction system when $R$ is $10 \mathrm{~K} \Omega$ (a), $50 \mathrm{~K} \Omega$ (b), $100 \mathrm{~K} \Omega$ (c), and $150 \mathrm{~K} \Omega$ (d).

directions. Visibly, larger $R$ is beneficial for outputting higher voltages within limit. Meanwhile, with $R$ further increasing, the output voltage will not show noticeable variation.
Additionally, the output voltage amplitudes and average generated electric power versus $R$ in both directions are illustrated in Figure 16. The average generated electric power is evaluated by the following equation [63]: 


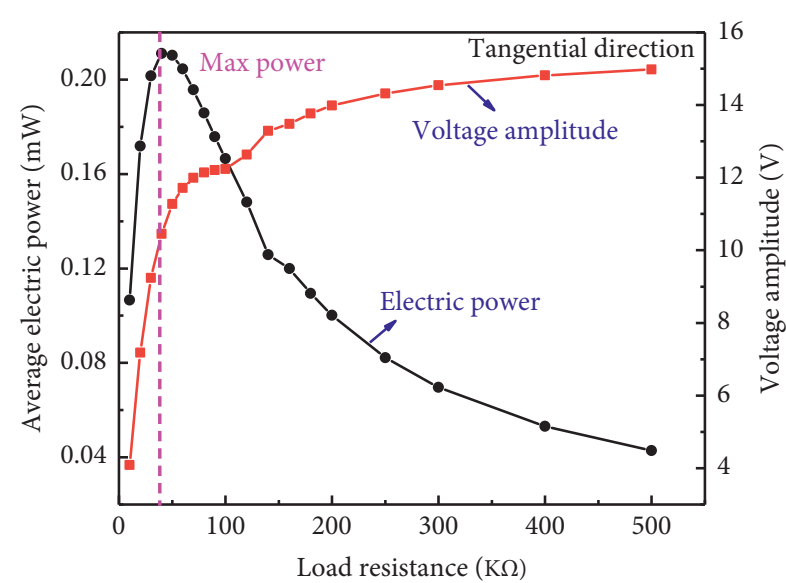

(a)

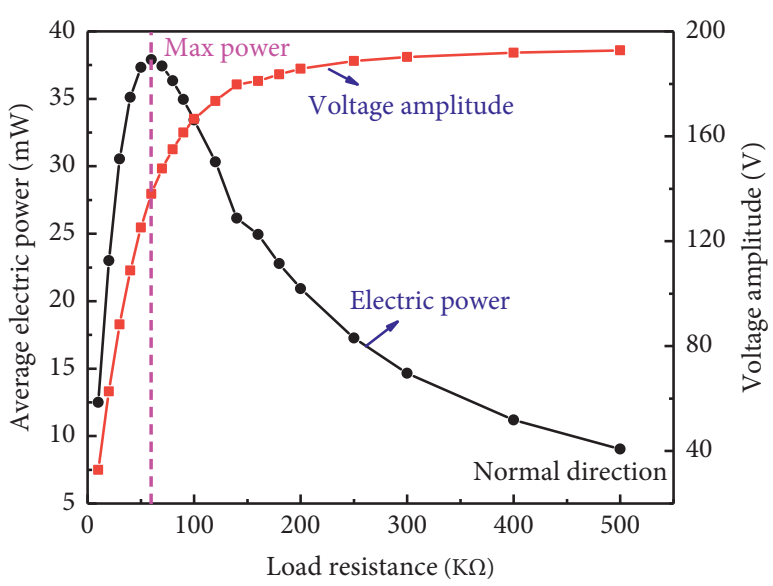

(b)

FIGURE 16: The output voltage amplitude and electric power of friction system with different $R$ in the tangential direction (a) and normal direction (b).

$$
P_{\text {average }}=\frac{\left(\int_{0}^{T}\left(V_{R}^{2} / R\right) \mathrm{d} t\right)}{T} .
$$

For the curve of output voltage amplitude (red line), it increases gradually at first and then remains stable basically with the increase of $R$. Meanwhile, for the electric power generated from the piezo elements, the curves (black line) show a trend of rising firstly and then falling with the further increase of $R$, indicating that there is an optimal external resistance that gives the maximum output power, which is in agreement with the experimental results reported by Wang et al. [8]. Moreover, it is worth noting that the external resistance of $40 \mathrm{~K} \Omega$ in the tangential direction is beneficial for the system to obtain the maximum average output power of $0.2112 \mathrm{~mW}$, while the external resistance of $60 \mathrm{~K} \Omega$ in the normal direction gives the maximum average output power of $38 \mathrm{~mW}$, which indicates that the optimal external resistance to generate highest power in the tangential direction is relatively less than that in the normal direction. The reason is that the output voltage in the tangential direction can arrive at a stable value at a lower optimal external resistance, compared to that of the output voltage in the normal direction. Therefore, it can be concluded that $R$ has little effect on the system's dynamical behaviors; however, it can significantly affect the energy harvesting behavior in a certain degree, and appropriate external electric resistances values in both directions are beneficial for obtaining the highest harvested energy.

\section{Conclusions}

A 2-DOF friction system (slider-on-belt model) with piezoelectric elements, which accounts for the stick, slip, separation, and reattachment between contact pairs, is employed to study the energy harvesting performance via friction-induced vibration (FIV). Both the eigenvalues analysis and transient dynamic analysis are carried out to study the dynamics and energy harvesting behavior of this friction system, and the main conclusions are summarized as follows:
(1) Eigenvalue analysis results show that friction coefficient value $\left(\mu_{c}=0.85\right)$ is the critical friction coefficient of the system which will generate selfexcited vibration. Transient dynamic analysis results verify the feasibility of piezoelectric energy harvesting $(\mathrm{PEH})$ by means of FIV. A larger friction coefficient is proved to be able to generate more intense vibration and output higher voltage.

(2) The dynamic and output voltages are crucially dependent on the relationship among the kinetic friction coefficient $\mu_{k}$, static friction coefficient $\mu_{s}$, and critical friction coefficient $\mu_{k}$. When the friction coefficient locates in the range of $\mu_{c}<\mu_{k}<\mu_{s}$, the system will generate stronger vibration and output higher voltage. In contrast, lower vibration and less voltage will be generated when the friction coefficient locates in the range of $\mu_{k}<\mu_{s}<\mu_{c}$.

(3) If the separation between contact pair is ignored, it is likely to overestimate or underestimate the vibration magnitude and output voltage amplitude. The overestimation or underestimation phenomenon is mainly determined by the located range of friction coefficient. These are very interesting findings and can be exploited in design of energy harvester by means of FIV.

(4) The external resistance $R$ has little effect on the dynamical behaviors of the friction system, but this will affect the output voltages of friction system within limits. In addition, there exists an appropriate value of $R$ for achieving the largest output voltage and electric power.

\section{Data Availability}

The data used to support the findings of this study are included within the article. 


\section{Conflicts of Interest}

The authors declare no conflicts of interest with respect to the research, authorship, and/or publication of this article.

\section{Acknowledgments}

This research received financial support from the National Natural Science Foundation of China (no. 51505396).

\section{References}

[1] S. Priya, H. C. Song, Y. Zhou et al., "A Review on piezoelectric energy harvesting: materials, methods, and circuits Energy," Energy Harvesting and Systems, vol. 4, no. 1, pp. 3-39, 2017.

[2] S. Sudevalayam and P. Kulkarni, "Energy harvesting sensor nodes: survey and implications," IEEE Communications Surveys \& Tutorials, vol. 13, no. 3, pp. 443-461, 2011.

[3] S. Priya, D. J. Inman, and J. Daniel, Energy Harvesting Technologies, Springer, New York, NY, USA, 2008.

[4] N. G. Stephen, "On energy harvesting from ambient vibration," Journal of Sound and Vibration, vol. 293, no. 1-2, pp. 409-425, 2006.

[5] H. S. Kim, J.-H. Kim, and J. Kim, "A review of piezoelectric energy harvesting based on vibration," International Journal of Precision Engineering and Manufacturing, vol. 12, no. 6, pp. 1129-1141, 2011.

[6] C. Wei and X. Jing, "A comprehensive review on vibration energy harvesting: modelling and Realization," Renewable and Sustainable Energy Reviews, vol. 74, pp. 1-18, 2017.

[7] R. Ahmed, F. Mir, and S. Banerjee, "A review on energy harvesting approaches for renewable energies from ambient vibrations and acoustic waves using piezoelectricity," Smart. Mater. Struct., vol. 26, no. 8, Article ID 085031, 2017.

[8] D. W. Wang, J. L. Mo, X. F. Wang, H. Ouyang, and Z. R. Zhou, "Experimental and numerical investigations of the piezoelectric energy harvesting via friction-induced vibration," Energy Conversion and Management, vol. 171, pp. 1134-1149, 2018.

[9] S. P. Beeby, R. N. Torah, M. J. Tudor et al., "A micro electromagnetic generator for vibration energy harvesting," Journal of Micromechanics and Microengineering, vol. 17, no. 7, pp. 1257-1265, 2007.

[10] F. U. Khan and M. U. Qadir, "State-of-the-art in vibrationbased electrostatic energy harvesting," Journal of Micromechanics and Microengineering, vol. 26, no. 10, Article ID 103001, 2016.

[11] A. Ertuk and D. J. Inman, Piezoelectric Energy Harvesting, John Wiley \& Sons, Chichester, U.K., 2011.

[12] L. Wang and F. G. Yuan, "Vibration energy harvesting by magnetostrictive material," Smart Materials and Structures, vol. 17, no. 4, pp. 1-10, 2008.

[13] J. Chen and Z. L. Wang, "Reviving vibration energy harvesting and self-powered sensing by a triboelectric nanogenerator," Joule, vol. 1, no. 3, pp. 480-521, 2017.

[14] Y. Fu, H. Ouyang, and R. B. Davis, "Triboelectric energy harvesting from the vibro-impact of three cantilevered beams," Mechanical Systems and Signal Processing, vol. 121, pp. 509-531, 2019.

[15] A. Erturk and D. J. Inman, "On mechanical modeling of cantilevered piezoelectric vibration energy harvesters," Journal of Intelligent Material Systems and Structures, vol. 19, no. 11, pp. 1311-1325, 2008.
[16] A. Erturk and D. J. Inman, "An experimentally validated bimorph cantilever model for piezoelectric energy harvesting from base excitations," Smart Materials and Structures, vol. 18, Article ID 025009, 2009.

[17] M. N. Fakhzan and A. G. A. Muthalif, "Harvesting vibration energy using piezoelectric material: modeling, simulation and experimental verifications," Mechatronics, vol. 23, no. 1, pp. 61-66, 2013.

[18] M. Kim, J. Dugundji, and B. L. Wardle, "Efficiency of piezoelectric mechanical vibration energy harvesting," Smart Materials and Structures, vol. 24, no. 5, Article ID 055006, 2015.

[19] J. Wang, S. Zhou, Z. Zhang, and D. Yurchenko, "High-performance piezoelectric wind energy harvester with Y-shaped attachments," Energy Conversion and Management, vol. 181, no. 1, pp. 645-652, 2019.

[20] H. C. Liu, J. W. Zhong, C. K. Lee, S. W. Lee, and L. W. Lin, “A comprehensive review on piezoelectric energy harvesting technology: materials, mechanisms, and applications," Applied Physics Reviews, vol. 5, Article ID 041306, 2018.

[21] V. R. Challa, M. G. Prasad, and F. T. Fisher, "Towards an autonomous self-tuning vibration energy harvesting device for wireless sensor network applications," Smart Materials and Structures, vol. 20, no. 2, pp. 1-11, 2011.

[22] M. Safaei, H. A. Sodano, and S. R. Anton, "A review of energy harvesting using piezoelectric materials: state-of-the-art a decade later (2008-2018)," Smart Materials and Structures, vol. 28, no. 11, Article ID 113001, 2019.

[23] X. Wang, C. Chen, N. Wang et al., "A frequency and bandwidth tunable piezoelectric vibration energy harvester using multiple nonlinear techniques," Applied Energy, vol. 190, pp. 368-375, 2017.

[24] H.-C. Song, P. Kumar, R. Sriramdas et al., "Broadband dual phase energy harvester: vibration and magnetic field," Applied Energy, vol. 225, pp. 1132-1142, 2018.

[25] J. Zhang, Z. Fang, C. Shu, J. Zhang, Q. Zhang, and C. Li, “A rotational piezoelectric energy harvester for efficient wind energy harvesting," Sensors and Actuators A: Physical, vol. 262, no. 1, pp. 123-129, 2017.

[26] H. Farokhi, A. Gholipour, and M. H. Ghayesh, "Efficient broadband vibration energy harvesting using multiple piezoelectric bimorph," Journal of Applied Mechanics, vol. 87, no. 4, Article ID 041001, 2020.

[27] L. Tang, Y. Yang, and C. K. Soh, "Toward broadband vibration-based energy harvesting," Journal of Intelligent $\mathrm{Ma}$ terial Systems and Structures, vol. 21, no. 18, pp. 1867-1897, 2010.

[28] S. Zhou, J. Cao, A. Erturk, and J. Lin, "Enhanced broadband piezoelectric energy harvesting using rotatable magnets," Applied Physics Letters, vol. 102, no. 17, Article ID 173901, 2013.

[29] Z. Yang, L. Tang, K. Tao, and K. Aw, “A broadband electretbased vibrational energy harvester using soft magneto-sensitive elastomer with asymmetrical frequency response profile," Smart Materials and Structures, vol. 28, no. 10, Article ID 10LT02, 2019.

[30] A. Erturk and D. J. Inman, "Broadband piezoelectric power generation on high-energy orbits of the bistable duffing oscillator with electromechanical couplingfing oscillator with electromechanical coupling," Journal of Sound and Vibration, vol. 330, no. 10, pp. 2339-2353, 2011.

[31] J. Yang, J. Chen, Y. Yang et al., "Broadband vibrational energy harvesting based on a triboelectric nanogenerator," Advanced Energy Materials, vol. 4, no. 6, p. 1301322, 2014. 
[32] S. Zhou, J. Cao, D. J. Inman, J. Lin, S. Liu, and Z. Wang, "Broadband tristable energy harvester: modeling and experiment verificationfication," Applied Energy, vol. 133, pp. 3339, 2014.

[33] X. Wang and L. W. Lin, "Dimensionless optimization of piezoelectric vibration energy harvesters with different interface circuits," Smart Materials and Structures, vol. 22, no. 8, Article ID 085011, 2013.

[34] Y. C. Shu and I. C. Lien, "Efficiency of energy conversion for a piezoelectric power harvesting systemficiency of energy conversion for a piezoelectric power harvesting system," Journal of Micromechanics and Microengineering, vol. 16, no. 11, pp. 2429-2438, 2006.

[35] S. Dhote, Z. Yang, K. Behdinan, and J. Zu, "Enhanced broadband multi-mode compliant orthoplanar spring piezoelectric vibration energy harvester using magnetic force," International Journal of Mechanical Sciences, vol. 135, pp. 63-71, 2018.

[36] J. Twiefel and H. Westermann, "Survey on broadband techniques for vibration energy harvesting," Journal of Intelligent Material Systems and Structures, vol. 24, no. 11, pp. 1291-1302, 2013.

[37] W. J. Ding, Self-Excited Vibration, Springer, Heidelberg, Germany, 2010

[38] R. A. Ibrahim, "Friction-induced vibration, chatter, squeal, and chaos-Part I: mechanics of contact and friction," Applied Mechanics Reviews, vol. 47, no. 7, pp. 209-226, 1994.

[39] R. A. Ibrahim, "Friction-induced vibration, chatter, squeal, and chaos-part II: dynamics and modeling," Applied Mechanics Reviews, vol. 47, no. 7, pp. 227-253, 1994.

[40] C. Cantoni, R. Cesarini, G. Mastinu, G. Rocca, and R. Sicigliano, "Brake comfort - a review," Vehicle System Dynamics, vol. 47, no. 8, pp. 901-947, 2009.

[41] J. Kang and K. Kim, "Squeak noise in lead screw systems: selfexcited vibration of continuous model," Journal of Sound and Vibration, vol. 329, no. 17, pp. 3587-3595, 2010.

[42] J. Le Rouzic, A. Le Bot, J. Perret-Liaudet et al., "Frictioninduced vibration by stribeck's law: application to wiper blade squeal noise," Tribology Letters, vol. 49, no. 3, pp. 563-572, 2013.

[43] N. M. Ghazaly, M. El-Sharkawy, and I. Ahmed, "A review of automotive brake squeal mechanisms," Journal of Mechanical Design, vol. 1, no. 1, pp. 5-9, 2014.

[44] J. J. Sinou and L. Jezequel, "On the stabilizing and destabilizing effects of damping in a non-conservative pin-disc system," Acta. Mechanica, vol. 199, no. 1-4, pp. 43-52, 2008.

[45] V.-V. Lai, O. Chiello, J.-F. Brunel, and P. Dufrénoy, "Full finite element models and reduction strategies for the simulation of friction-induced vibrations of rolling contact systems," Journal of Sound and Vibration, vol. 444, pp. 197-215, 2019.

[46] F. Massi, L. Baillet, and A. Culla, "Structural modifications for squeal noise reduction: numerical and experimental validation," International Journal of Vehicle Design, vol. 51, no. 1-2, pp. 168-189, 2009.

[47] R. Ariyatanapol, Y. P. Xiong, and H. Ouyang, "Partial pole assignment with time delays for asymmetric systems," Acta Mechanica, vol. 229, no. 6, pp. 2619-2629, 2018.

[48] M. Stender, M. Tiedemann, and N. Hoffmann, "Energy harvesting below the onset of flutter," Journal of Sound and Vibration, vol. 458, pp. 17-21, 2019.

[49] J.-J. Sinou and L. Jézéquel, "Mode coupling instability in friction-induced vibrations and its dependency on system parameters including damping," European Journal of Mechanics - A/Solids, vol. 26, no. 1, pp. 106-122, 2007.

[50] K. Shin, M. J. Brennan, J.-E. Oh, and C. J. Harris, "Analysis of disc brake noise using a two-degree-of-freedom model," Journal of Sound and Vibration, vol. 254, no. 5, pp. 837-848, 2002.

[51] K. Popp and P. Stelter, "Stick-slip vibrations and chaos," Philosophical Transactions of the Royal Society of London. Series A: Physical and Engineering Sciences, vol. 332, no. 1624, pp. 89-105, 1990.

[52] L. E. Helseth, "Excitation of energy harvesters using stick-slip motion," Smart Materials and Structures, vol. 23, Article ID 085024, 2014.

[53] C. Tadokoro, A. Matsumoto, T. Nagamine, and S. Sasaki, "Piezoelectric power generation using friction-induced vibration," Smart Materials and Structures, vol. 26, Article ID 065012, 2017.

[54] A. Masuda and C. Sawai, "Stick-slip energy harvesting: a preliminary study," in ASME 2017 Conference on Smart Materials, Adaptive Structures and Intelligent Systems, Snowbird, Utah, USA, Septamber 2017.

[55] E. Heffel and P. Hagedorn, "Friction induce vibration for energy harvesting applications," IWPMA/EHW Hannover, vol. 14, 2013.

[56] D. W. Wang, M. X. Liu, W. J. Qian, X. Wu, Q. Ma, and Z. Q. Wu, "Parametrical investigation of piezoelectric energy harvesting via friction-induced vibration," Shock and Vibration, vol. 2020, pp. 1-32, 2020.

[57] Z. L. Li, H. Ouyang, and Z. Q. Guan, "Nonlinear frictioninduced vibration of a slider-belt system," Journal of Vibration and Acoustics, vol. 138, Article ID 041006, 9 pages, 2016.

[58] A. Niknam and K. Farhang, "Friction-induced vibration due to mode-coupling and intermittent contact loss," Journal of Vibration and Acoustics, vol. 141, no. 2, Article ID 021012, 2019.

[59] A. Medio and M. Lines, Nonlinear Dynamics: A Primer, Cambridge University Press, Cambridge, UK, 2001.

[60] N. Hoffmann, M. Fischer, R. Allgaier, and L. Gaul, "A minimal model for studying properties of the mode-coupling type instability in friction induced oscillations," Mechanics Research Communications, vol. 29, no. 4, pp. 197-205, 2002.

[61] K. Soobbarayen, J.-J. Sinou, and S. Besset, "Numerical study of friction-induced instability and acoustic radiation - effect of ramp loading on the squeal propensity for a simplified brake modelfied brake model," Journal of Sound and Vibration, vol. 333, no. 21, pp. 5475-5493, 2014.

[62] J. Brunetti, F. Massi, W. D’Ambrogio, and Y. Berthier, "Dynamic and energy analysis of frictional contact instabilities on a lumped system," Meccanica, vol. 50, no. 3, pp. 633-647, 2015.

[63] B. A. M. Owens and B. P. Mann, "Linear and nonlinear electromagnetic coupling models in vibration-based energy harvesting," Journal of Sound and Vibration, vol. 331, no. 4, pp. 922-937, 2012. 